IMA Journal of Applied Mathematics (2020) Page 1 of 30

doi:10.1093/imamat/xxx000

\title{
Detachment in capillary adhesion: The relative roles of tilting and separation
}

\author{
Matthew D. Butler And Dominic Vella \\ Mathematical Institute, University of Oxford, Woodstock Rd, Oxford OX2 6GG, United \\ Kingdom
}

[Received on 3 July 2020]

\begin{abstract}
We study the dynamics of detachment in two-dimensional capillary adhesion by considering a plate that is initially attached to a flat, rigid substrate via the surface tension of a bridging liquid droplet. In particular, we focus on the effect of allowing the plate to tilt freely during its subsequent motion. A linear stability analysis shows that small perturbations from equilibrium decouple into two modes: one in which the plate separates from the substrate, remaining parallel, and another in which it tilts, simultaneously causing the bridging droplet to migrate. If the initial tilt perturbation is of a similar magnitude to (or bigger than) the separation perturbation, then the presence of this second, tilting mode can significantly alter the dynamics. Indeed, this tilting mechanism changes the ultimate fate of the plate: depending on the size of the plate and the initial perturbation, the plate may anomalously detach. We discuss this observation in relation to previous experiments on a three-dimensional system that showed a qualitatively similar anomalous detachment.
\end{abstract}

Keywords: Surface tension, capillary adhesion, detachment, lubrication theory

2000 Math Subject Classification: 76D08, 76D45, 37N10

\section{Introduction}

The ability to attach two surfaces (and subsequently detach them) under an applied load is important in many everyday, industrial and biological situations. Alongside the more common methods of attachment - such as glues, welds and claws or grippers - recent scientific study has opened up new avenues for potential adhesive mechanisms. In particular, examples of adhesion seen in nature have been the inspiration behind many novel man-made adhesives, with examples including 'Gecko tape' (see Geim et al., 2003; Gorb et al., 2007, for example) and the snail-inspired hydrogel adhesive of Cho et al. (2019).

In developing such adhesives it is important not only to understand how they initially adhere, but also how detachment occurs. This gives an insight into how the adhesion fails, giving the strength of the adhesive, and is particularly important when developing reusable adhesives: understanding how adhesives can be detached efficiently and controllably is key to repeatable adhesion.

One particularly interesting biological case is the adhesion of insects - they are able to stick to (and un-stick from) apparently smooth surfaces, such as glass, and to support loads that are many times their weight (Dirks \& Federle, 2011). While it is believed that many insects use the surface tension of a liquid to perform this adhesion (Dirks, 2014), many details, including how insects control 'capillary adhesion', remain unclear.

When a fluid droplet is confined between two solids, it applies a force on them due to the surface tension that acts at the interface between the fluid and the surrounding environment. These capillary effects can be substantial, particularly at microscopic scales (Lambert et al., 2013) where surface tension forces 
(which scale with length) dominate volume forces such as gravity (which scale with length cubed). This observation suggests that capillary systems may be a particularly fruitful area for study when designing small-scale adhesive devices (such as the switchable capillary adhesive of Vogel \& Steen, 2010).

BASIC MODEL OF CAPILLARY ADHESION The presence of a capillary bridge between two rigid, planar surfaces is often used as a basic case through which to understand capillary adhesion in more complicated circumstances. This scenario is shown in fig. 1a: a liquid droplet of volume $V$ bridges the gap (of width $h$ ) between two parallel rigid surfaces. We will consider one of these surfaces, which we call the substrate, to always be fixed in place and consider how the other solid may be attached to the substrate using the surface tension of the droplet.The interface of the droplet meets the rigid surfaces at an angle $\theta$, the contact angle, that can be determined by the Young-Dupré equation (see, for example, Chapter 1.2 of de Gennes et al., 2004). This contact angle condition forces the droplet surface to become curved.

Surface tension forces are present whenever there is an interface between two immiscible fluids. This surface tension acts tangentially to the interface, causing a jump in fluid pressure across the interface that is proportional to the interface curvature: $\Delta p=\gamma\left(1 / R_{1}+1 / R_{2}\right)$. Here, $\gamma$ is the surface tension and the $R_{i}$ are the principal radii of curvature of the surface. In this way, the shape of an interface determines the pressure jump between the two fluids.

The pressure difference between the droplet and the atmosphere, $\Delta p$, acts over the area of the fluid footprint, $A$, thereby applying a net pressure force $\Delta p \times A$. The sign of this pressure force itself depends on the wetting properties of the droplet. A non-wetting droplet (contact angle $\theta>\pi / 2$ ) has two positive principal curvatures, and hence a positive internal pressure resulting in a repulsive force. Wetting droplets $(\theta<\pi / 2)$, however, have two curvatures of different sign; when the droplet is much wider than it is tall, it has a negative capillary pressure and hence exerts an attractive force between the two solids, which is the capillary adhesion of interest here.

In addition, a tension force $\gamma$ per unit length of the contact line acts in a direction parallel to the interface. This contributes a force $\gamma \sin \theta \times b$, with $b$ the perimeter of the droplet footprint. Note that this contact line force is always attractive and hence that the forces due to the contact line and the pressure within the droplet act in opposing directions for non-wetting droplets $(\theta>\pi / 2)$; we are interested in capillary adhesion (i.e. attractive forces) and so focus on the case of wetting droplets $(\theta<\pi / 2)$ here.

In general, the problem of determining the equilibrium shape of the droplet between two planar surfaces requires the solution of a boundary value problem for a surface of constant curvature (such as the work of Lowry \& Steen, 1995) or the minimization of an energy functional (as studied by Carter, 1988 , for example). For very short and wide droplets, the picture simplifies since the pressure within the droplet is given to a very good approximation by $\Delta p \approx-2 \gamma \cos \theta / h$ (the error is a factor $h / R$ smaller, with $R$ the droplet radius - see Reyssat, 2015, for example) and the tension component of the force provides a negligible contribution if the fluid is sufficiently wetting $(\tan \theta \ll R / h)$. Moreover, since the area of contact of the droplet is $A \approx V / h$, we then have that the magnitude of the adhesive force is

$$
F=\frac{2 \bar{\gamma} V}{h^{2}}
$$

where we define $\bar{\gamma}=\gamma \cos \theta$. Note that if the contact angle differs on each surface, then we can instead use the average of the two cosines: $\bar{\gamma}=\gamma\left(\cos \theta_{1}+\cos \theta_{2}\right) / 2$.

For a given applied load, $F=W$, and given droplet properties (i.e. given $V$ and $\bar{\gamma}$ ), (1.1) gives an equation for the equilibrium displacement of the planar surfaces that would provide sufficient force to support that load. The question then arises of whether this equilibrium is stable. 
(a)

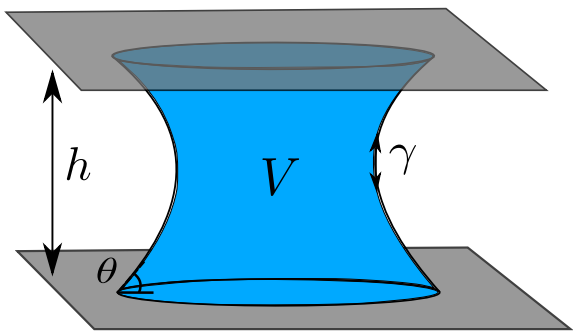

(b)

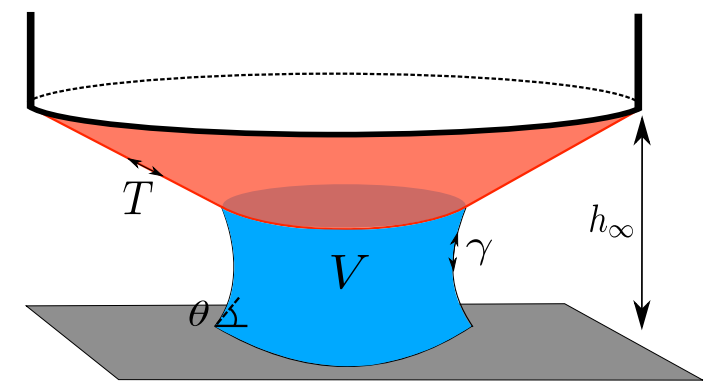

FIG. 1. (a) Two rigid planar surfaces are adhered together by the surface tension of a fluid droplet. (b) A fluid droplet adheres a tense membrane to a rigid planar surface. The membrane is circular and clamped at the edges. Figure in (b) reprinted with permission from Butler et al. (2019). Copyright (2019) by the American Physical Society.

SIMPLE CAPILLARY ADHESION IS UNSTABLE UNDER FIXED LOAD The stability of an axisymmetric droplet supporting a given planar load was studied previously by Ward (2011). In particular, by modelling the motion of the liquid within the droplet using lubrication theory, Ward (2011) derived an ordinary differential equation (ODE) for the evolution of the separation between a substrate and a mobile plate and found that the above equilibrium (1.1) is linearly unstable to small perturbations. The analysis of Ward (2011) showed that perturbations that act to decrease the plate-substrate separation (relative to the equilibrium value) ultimately lead to contact between the two planar surfaces; perturbations that increase this separation ultimately lead to further separation (though the ultimate dynamics of rupture of the liquid bridge were not considered).

Experiments supported this general picture, although Ward (2011) also found that, in some cases, small negative perturbations in the plate-substrate separation (which would be expected to lead to contact between plate and substrate) ultimately led to complete separation (see fig. 5a). Ward (2011) suggests that this small discrepancy could be attributed to some experimental inaccuracies or the assumption of perfect wettability. However, in this paper we investigate the possibility that relative tilting of the plate compared to the substrate (which was neglected by Ward, 2011) might in fact lead to a similar behaviour: we consider a two-dimensional model of the dynamics of a loaded plate attached to a substrate by the capillary force of a droplet, including the effects of tilting and translation on the plate, as well as allowing the droplet to move. (Such motion is expected to occur whenever tilting leads to tapering, as studied previously by Reyssat (2014).) This study is also motivated by experimental observations of the failure of another kind of capillary adhesive (Butler et al., 2019), and so we first describe that problem in more detail.

\section{Capillary adhesion and detachment of a deformable membrane}

In a previous paper (Butler et al., 2019), we considered the capillary adhesion caused by a single droplet, similar to fig. 1a, but with one of the rigid surfaces replaced by a deformable membrane. In this problem, a thin circular membrane is clamped around its edges whilst subject to an applied tension $T$, and the edge of the membrane is maintained at a height $h_{\infty}$ above a rigid, planar substrate. This set-up is shown schematically in fig. $1 \mathrm{~b}$.

In contrast to the rigid-walled capillary adhesion described in the introduction, the presence of a 
membrane deformability (controlled by the applied tension $T$ ) in addition to the plate separation, $h_{\infty}$, gives rise to different behaviour, and new routes for the control of adhesion. As might be expected, for sufficiently large applied tensions the membrane is not so deformable and the observed adhesion force follows that predicted in (1.1). However, for sufficiently weak tensions (or, equivalently, $h_{\infty}$ small enough to cause a large capillary pressure and hence some deformation) a qualitatively different solution emerges in which the two solid surfaces are in contact over some region. Crucially, in this contacting equilibrium, the adhesion force can be orders of magnitude larger than that expected on the basis of (1.1), for which there is no contact. These predictions of a mathematical model are supported by experiments conducted on a lab scale realization of the model system, with measurements of the force-separation curves showing good agreement without any fitting parameters. Moreover, the applied tension may be increased after an object has adhered, allowing the adhesion to be decreased (and the load released) in a controllable way, and without the need to 'yank' the materials apart.

(a)

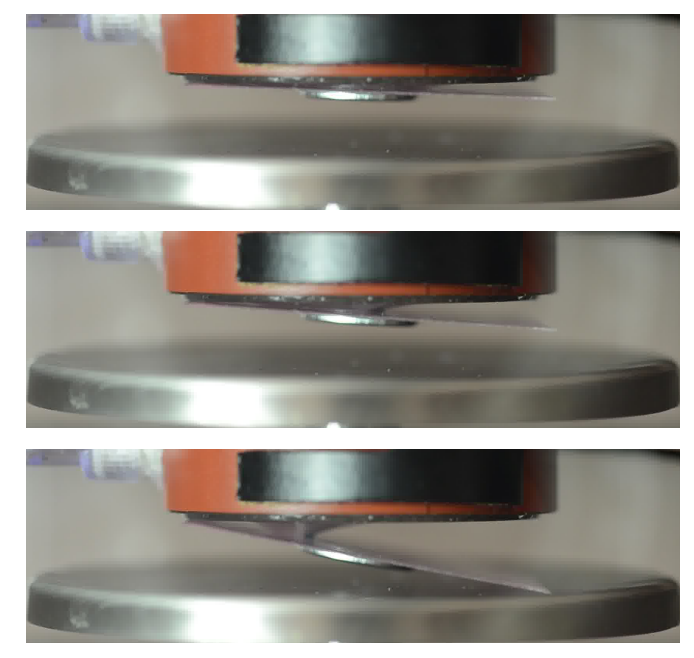

(b) Rigid

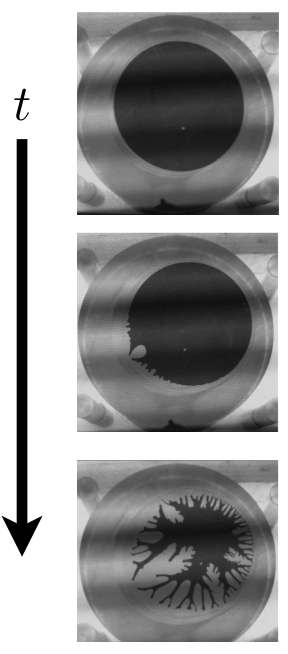

FIG. 2. Evidence for asymmetric detachment in previous experiments. (a) In a deformable adhesive, after a transient adhesion, the load detaches by peeling off from one edge. Images courtesy of Finn Box. (b) Plan view of the fluid remaining when a rigid capillary adhesive detaches. In the second image, we see evidence of detachment beginning towards the bottom left corner of the droplet. Images in (b) reprinted from Ward (2011), with permission from Elsevier.

This deformable capillary adhesive was found to be able to pick up and release loads in a similar manner to that expected from the theory. However, quantitative comparisons between the load supported and the various control parameters in the system showed that loads often detached even when the equilibrium theory suggested that strong adhesion should be achieved. Butler et al. (2019) suggested that this is partly due to the long time taken for the deformable surface to 'snap' into contact with the rigid surface - a delay that is caused by the formation of a fluid dimple beneath the membrane as it is pulled towards contact with the substrate. The viscous fluid in the dimple drains out of this dimple slowly, slowing the approach to equilibrium, meaning that in reality lasting adhesion may only be achieved if the two surfaces are left for some time to adhere. This explanation is consistent with the observation 
that loads adhere indefinitely if left in contact for sufficiently long times.

However, observations of how the load detaches in unsuccessful adhesion experiments reveals that this detachment propagates from one edge, as shown in the snapshots in fig. 2a. The axisymmetric theory of Butler et al. (2019) is unable to reproduce this scenario and so, when trying to predict the detachment time, we overestimate how long the load would stay attached. A similar asymmetry is also visible in snapshots of the experiments of Ward (2011): fig. $2 \mathrm{~b}$ shows evidence that at least some experiments detach towards one edge. This suggests that this asymmetric detachment mechanism may be more universal than just for the deformable case, and hence warrants further study.

\section{Detachment of a loaded plate in two dimensions}

To understand the possible importance of tilting in detachment, rather than simply detaching by separating, we consider a simplified model of detachment in capillary adhesion: a two-dimensional droplet of volume $V$ (per unit depth into the page) is confined between a rigid substrate and a rigid plate of length $2 \ell$. This scenario is illustrated in fig. 3. The substrate is flat and fixed in place, whilst the plate has a load (per unit length) $W$ applied at its centre of mass. (This load could be the weight of the plate, for example.) In general, we shall assume that the plate is free to separate from the substrate, rotate relative to it, and translate parallel to the substrate. These motions are defined by the separation $h$ between the centre of mass of the loaded plate and the substrate, the angle $\alpha$ between them, and the horizontal speed $u$ of the plate relative to the substrate.

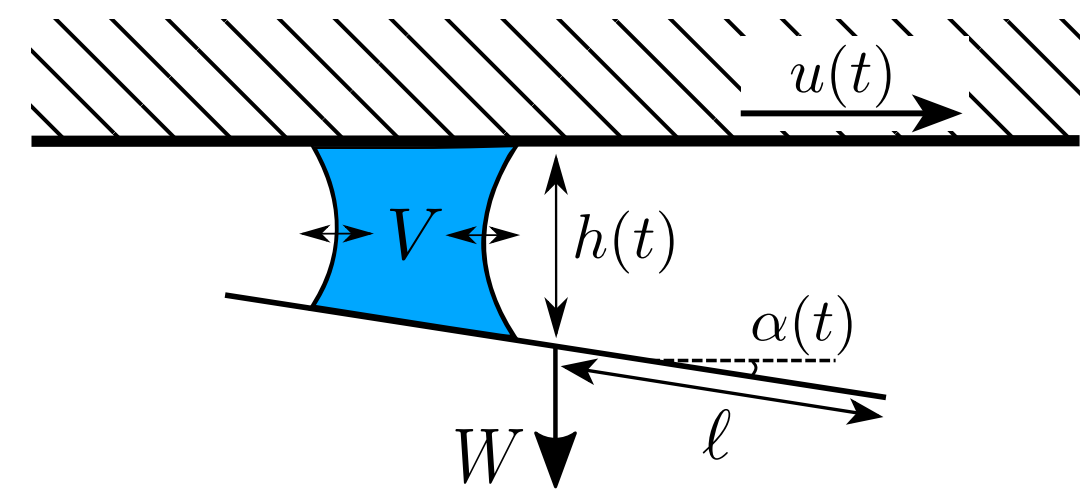

FIG. 3. The surface tension of a two-dimensional droplet of volume $V$ adheres a mobile plate of length $2 \ell$ subject to an applied load (per unit depth) $W$ to a fixed planar substrate. The mobile plate makes an angle $\alpha(t)$ to the substrate and its centre of mass lies a vertical distance $h(t)$ below the substrate. We work in the frame of reference of the (mobile) plate, so that the top surface may move relative to the plate at a speed $u(t)$.

As the plate separates from the substrate and rotates, the change in geometry that the droplet experiences will alter the capillary pressure, and the fluid flow will give an additional viscous forcing. Ignoring the effect of gravity on the droplet (since the Bond number of such droplets is typically small), the combination of these two forces will determine the motion of the plate and droplet together.

The possibility that the plate may tilt is important since the geometry of tilting, together with the pressure jump across a meniscus, means that as soon as the plate tilts the capillary pressure 'at' the two menisci must differ. For wetting droplets (as considered here), such a tapering-induced pressure difference will tend to drive the droplet towards the narrower end, as was first observed by Hauksbee 
(1710). This confinement-induced migration has been studied quantitatively recently via simulations (Concus \& Finn, 1998; Ruiz-Gutiérrez et al., 2018; Ruiz-Gutiérrez \& Ledesma-Aguilar, 2020) and experimentally (Renvoisé et al., 2009; Reyssat, 2014; Bradley et al., 2019). (For non-wetting drops, motion occurs towards the wider end in $2 \mathrm{D}$, while in 3D droplet equilibria are possible, provided that the surface is sufficiently non-wetting, dependent on the wedge angle, as studied by Concus \& Finn, 1998; Concus et al., 2001; Ruiz-Gutiérrez et al., 2018, for example.)

A key effect of allowing the plate and substrate to tilt relative to one another is, therefore, that any tilting motion may be accompanied by droplet migration. Moreover, the asymmetry induced by a capillary pressure difference across the droplet, together with the induced motion of the droplet away from the centre of the plate, will, in general, apply a torque that acts to rotate the loaded plate even further - we expect the horizontal state of the plate to be unstable to rotation.

In addition, once the plate is tilted, the flow of fluid towards the narrower end due to the capillary pressure gradient will exert a viscous shear force on the plate, and a component of the adhering capillary pressure will act horizontally on the angled plate. The combination of these forces may lead to the plate drifting horizontally. Previous studies of rigid plates supported by thin fluid layers have found that an angled plate can migrate horizontally when sedimenting in a viscous bath towards a wall (Argentina et al., 2007), while Trinh et al. (2014) and Moriarty \& Terrill (1996) explored the steady state translation of a plate on the surface of a thin fluid film. It is possible that similar horizontal drift may be observed in our adhesion problem that will affect the evolution of the plate-droplet system, and so we include the possibility of this in our model as well.

To quantify our intuition about the rotational instability, we consider a droplet that is substantially wider than the plate separation. This means that we can ignore the effect of the capillary tension force from the pull of the meniscus on the plate at the droplet edge, since it is approximately a droplet aspect ratio smaller in size. Instead, we calculate the force solely from the capillary pressure acting over the wetted footprint of the droplet.

We start by considering the equilibrium of this system, before moving on to consider two simplified models: the first reprises the model of Ward (2011) (albeit in 2D) with no tilting of the plate (the angle $\alpha=0$ is fixed). In the second simplified model, the plate is considered to be fixed about a central hinge (i.e. constant $h$ with no horizontal translation) and able to rotate freely. This 'see-saw' motion allows us to isolate the effect of droplet migration and the concomitant torque. These two motions are then combined with the added effect of plate shearing in $\S 4$ into a full model in which the plate may slide parallel to, separate from, and rotate relative to, the substrate - the resulting model equations are solved numerically, but can also be understood using a linear stability analysis and by referring back to the simpler models considered in this section.

\subsection{Equilibrium}

When the system is in equilibrium, the plate and the fluid in the droplet are stationary and the menisci lie at $x= \pm x_{0}$; furthermore there is no pressure gradient. For this to be true, the curvature of the two free surfaces must be constant and equal, and so take the shape of arcs of circles with $\alpha=0$. Matching the contact angle on each side, these circular arcs have radius $h_{0} /(2 \cos \theta)$ with $h=h_{0}$ the equilibrium plate separation. The pressure inside the droplet is then $p=-2 \bar{\gamma} / h_{0}$, and this capillary pressure force must balance the load, i.e.

$$
W=\int_{-x_{0}}^{x_{0}}-p \mathrm{~d} x=\frac{4 \bar{\gamma} x_{0}}{h_{0}} .
$$


Hence, given a load $W$ and droplet volume $V=2 x_{0} h_{0}$, there is a unique equilibrium plate separation

$$
h_{0}=\sqrt{\frac{2 \bar{\gamma} V}{W}} .
$$

Note that there is therefore a simple relationship between the load applied and the aspect ratio of the fluid droplet, namely

$$
\frac{2 x_{0}}{h_{0}}=\frac{W}{2 \bar{\gamma}} .
$$

\subsection{Modelling the fluid dynamics}

We now consider the motion of the plate, which is coupled closely to the dynamics of the fluid in the droplet. Because the droplet is assumed to be much wider than the plate separation, $x_{0} / h_{0} \gg 1$, we use the lubrication approximation in which the fluid motion is mostly tangential to the substrate and viscosity dominates over fluid inertia.

Consistent with this, the plate angle (and hence the slope) must remain small, $\alpha \ll 1$, and we take the approximation $\tan \alpha \approx \alpha$ so that the separation of the plate from the substrate is given by $h(t)+\alpha(t) x$ along its length. Additionally, since the fluid inertia is negligible, we can choose in which Galilean frame of reference to view the problem. We consider the evolution of the system in the frame of motion of the plate; the substrate shears at a speed $u(t)$ in the $x$-direction with $h(t)$ being the separation at the plate's centre of mass at each point in time, as shown in fig. 3. The horizontal distances $x$ are measured with respect to the centre of mass, so that the plate has its ends at $x= \pm \ell \cos \alpha \approx \pm \ell$.

Using the no-slip boundary condition on the two surfaces together with conservation of mass in the standard manner (see Chapter 5 of Leal, 2007, for example) shows that the pressure $p(x, t)$ must obey Reynolds' equation

$$
\frac{\partial(h+\alpha x)}{\partial t}+\frac{\partial q}{\partial x}=0, \quad q=-\frac{(h+\alpha x)^{3}}{12 \mu} \frac{\partial p}{\partial x}+\frac{h+\alpha x}{2} u
$$

where $q$ is the depth-integrated volumetric flux and $\mu$ is the dynamic viscosity of the fluid in the droplet.

Integrating Reynolds' equation, (3.4), once with respect to $x$, and introducing an (unknown) integration constant $c=c(t)$, gives the pressure gradient

$$
\frac{\partial p}{\partial x}=6 \mu \frac{\dot{\alpha} x^{2}+2 \dot{h} x+c}{(h+\alpha x)^{3}}+\frac{6 \mu u}{(h+\alpha x)^{2}}
$$

where we use dots to denote derivatives with respect to time. Note that $c=-2 q_{0}$ is related to the volumetric flux across the midpoint of the plate, $q_{0}=q(x=0)$.

At the meniscus, the action of surface tension causes a jump in the normal stress proportional to the curvature of the interface. However, since we are using the lubrication approximation, the fluid pressure does not vary significantly between the solid surfaces, just along them (in the $x$-direction). Additionally, the normal stress at the meniscus is dominated by the fluid pressure there. Each meniscus must therefore have a constant curvature, and so takes the shape of a circular arc and, moreover, the fluid pressure there must obey the Young-Laplace equation

$$
p\left(x_{ \pm}\right)=\frac{-2 \bar{\gamma}}{h+\alpha x_{ \pm}}
$$


(a)

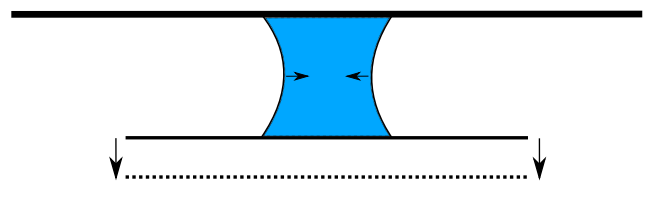

(b)

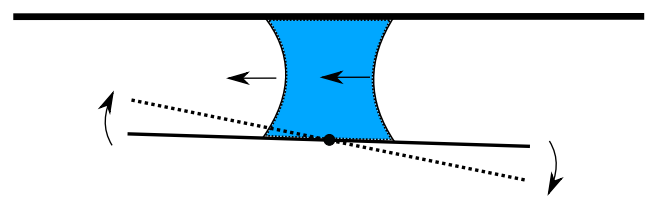

FIG. 4. The two modes through which the plate may move in relation to the substrate: (a) pure separation from the substrate (no tilting) and (b) pure tilting about a central hinge (the 'see-saw' mode). Note that the tilting mode induces a pressure gradient within the bridging droplet, which leads to the droplet itself migrating into the taper that is created by tilting.

where we have taken the external atmospheric pressure as the pressure datum, $p=0$.

To close the system, we require global relations for the motion of the plate, such as force and torque balances. Which relations are needed depends carefully on the set-up of the problem; we will now consider the two distinct motions shown in fig. 4 , and introduce the relevant global relations as they arise.

\subsection{Parallel plate: No tilting}

Consider the case where the plate does not tilt and remains parallel to the substrate, moving either directly towards or away from the substrate (without sliding by symmetry), as depicted in fig. 4a. In this case the plate position is given by $h=h(t)$ only, and the pressure gradient (3.5) can be simplified (eliminating the integration constant through the symmetry of the problem) to give

$$
\frac{\partial p}{\partial x}=12 \mu \frac{\dot{h} x}{h^{3}} .
$$

We impose a vertical force balance between the load and capillary force acting on the loaded plate

$$
W=\int_{x_{-}}^{x_{+}}-p \mathrm{~d} x=-[x p]_{\mathrm{drop}}+\int_{x_{-}}^{x_{+}} x \frac{\partial p}{\partial x} \mathrm{~d} x
$$

where $[f(x)]_{\text {drop }}=f\left(x_{+}\right)-f\left(x_{-}\right)$denotes the difference in the function $f(x)$ across the droplet. The pressure at each end is determined by the pressure jump due to capillary effects (3.6).

If the position of the droplet edges, or menisci, are given by $x_{ \pm}= \pm x_{M}$ then volume conservation immediately gives $V=2 x_{M} h$. Substituting (3.7) into (3.8), and using this volume conservation to eliminate $x_{M}$, gives the evolution equation for the plate separation

$$
\dot{h}=\frac{W}{\mu V^{3}}\left(h^{2}-h_{0}^{2}\right) h^{4}
$$

As expected, $h=h_{0}$ is an equilibrium of (3.9); moreover, (3.9) is similar to the form of the dimensionless equation found by Ward for the axisymmetric system (eqn. 3 of Ward, 2011). (In the axisymmetric case, the pre-factor and equilibrium solution are different, as well as there being one power fewer of $h$ than here.) It is easy to see from the non-linear ODE (3.9) that $\dot{h}<0$ whenever $0<h<h_{0}$ while $\dot{h}>0$ whenever $h>h_{0}$ : as discussed in the introduction the equilibrium $h=h_{0}$ is unstable and the plate will tend to contact the substrate for an initial perturbation with $h(0)<h_{0}$ or complete separation for an initial perturbation with $h(0)>h_{0}$. 
Considering a small perturbation from equilibrium $h(t)=h_{0}+\delta h(t)$ with $\delta h \ll h_{0}$ and linearizing, confirms that the equilibrium is linearly unstable and that the perturbation grows exponentially:

$$
\delta h(t)=\delta h(0) e^{t / t_{*}},
$$

over a time scale

$$
t_{*}=\frac{\mu V^{3}}{2 W h_{0}^{5}}=\frac{\mu x_{0}^{2}}{\bar{\gamma} h_{0}}
$$

Note that by assuming that viscous forces precisely balance the load $W$, as expressed in (3.8), we have neglected the inertia of the plate itself. This assumption is valid provided that the plate inertia, $m \ddot{h}$ (with $m$ the plate mass per unit length), is small compared to the pressure force and the applied load $W$. This means the plate inertia is unimportant over times $t$ such that $W \gg m h_{0} / t^{2}$; for inertia to be negligible over time scales comparable with $t_{*}$ in (3.11) we find that the load must, surprisingly, be sufficiently large and, in particular, satisfy $W / \bar{\gamma} \gg\left[m^{2} \bar{\gamma}^{2} /\left(\mu^{4} V\right)\right]^{1 / 9}$. In general, the load is independent of the weight of the plate, but in cases where the load is provided by the weight of the plate, $W=m g$ with $g$ the acceleration due to gravity, then this condition can be rewritten as $W / \bar{\gamma} \gg\left[\bar{\gamma}^{4} /\left(\mu^{4} g^{2} V\right)\right]^{1 / 7}$. From (3.3), these conditions for neglect of plate inertia correspond to sufficiently thin drops in equilibrium, consistent with our use of lubrication theory to model the fluid flow. (We note that for very early times, a temporal boundary layer is expected; for the large aspect ratio droplets of interest here, we expect this phase to be very brief and, hence, to have negligible effect on the subsequent dynamics considered here.)

In fig. 5b, we plot the evolution determined from equation (3.9) when starting from small perturbations to the equilibrium solution (dashed line). We see that any perturbation grows, as predicted by the linear stability analysis. Experiments on the three-dimensional system, however, appear to have a more complex behaviour (see fig. 5a): some loads detach when they would be expected to stick, i.e. they ultimately detach even when $h(0)<h_{0}^{3 D}=\sqrt{2 \bar{\gamma} V_{3 D} / W_{3 D}}$ where $h_{0}^{3 D}$ is the equilibrium solution for the three-dimensional system with an applied load $W_{3 D}$ and droplet volume $V_{3 D}$ (see eqn. (1.1)). We refer to this as 'anomalous detachment'. One aspect that may be present in the experimental system, but has not been taken into account in our modelling thus far, is any possible perturbation to the orientation of the plate. We turn to consider first the effect of this tilting in isolation.

\subsection{Hinged plate: Only tilting}

Now, instead, we consider a plate that is hinged at its centre $x=0$ and is free to rotate (but not translate), as shown in fig. $4 \mathrm{~b}$. For simplicity we will take the hinge to be at the equilibrium distance $h_{0}$ from the substrate. In this case, the plate separation is $h_{0}+\alpha x$ and (3.5) becomes

$$
\frac{\partial p}{\partial x}=6 \mu \frac{\dot{\alpha} x^{2}+c}{\left(h_{0}+\alpha x\right)^{3}}
$$

Since the position of the hinge here is fixed, there is no need to consider force balance on the tilting plate. Instead we note that the pressure gradient integrated across the droplet must match the capillaryenforced pressure at the edges, and we consider the torque balance (taken about the hinge) to determine 
(a)

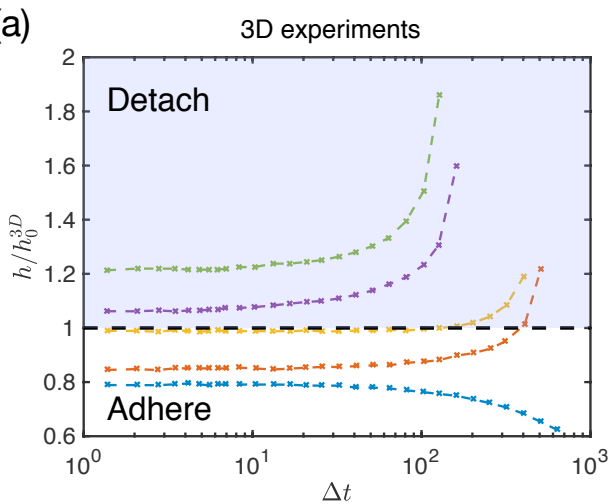

(b)

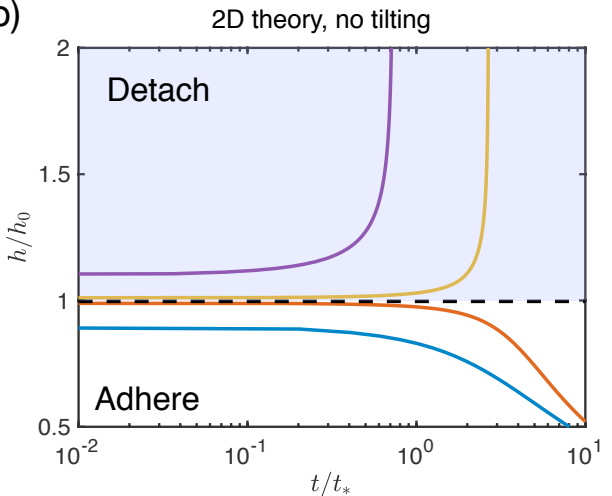

FIG. 5. Qualitative comparison of the evolution of the gap separation, $h(t)$, between three-dimensional experiments (Ward, 2011) and the simplest two-dimensional model in which the plate remains parallel to the substrate throughout the motion. (a) Experimental measurements of the separation of an acrylic mass from a fixed (acrylic) substrate resisted by a droplet of silicone oil over time, $\Delta t$, measured in seconds. Data digitally captured from fig. 6a of Ward (2011). The horizontal dashed line denotes the unstable equilibrium predicted within the axisymmetric theory, with $h_{0}^{3 D}$ given by (1.1). (b) Numerical solutions of (3.9), which describes the separation of the two-dimensional system with no tilting of the plate. In both plots the lightly shaded region corresponds to that in which a linear stability analysis suggests detachment should occur, while the unshaded region shows that in which the two surfaces should move towards contact - adhesion. Note that in (a) two experimental trajectories begin within the 'adhere' region but ultimately detach.

how the angle $\alpha$ changes. Together these conditions can be written as

$$
\begin{gathered}
-2 \bar{\gamma}\left[\left(h_{0}+\alpha x\right)^{-1}\right]_{\mathrm{drop}}=[p]_{\mathrm{drop}}=\int_{x_{-}}^{x_{+}} \frac{\partial p}{\partial x} \mathrm{~d} x, \\
0=\int_{x_{-}}^{x_{+}} x p \mathrm{~d} x=\frac{1}{2}\left[x^{2} p\right]_{\mathrm{drop}}-\frac{1}{2} \int_{x_{-}}^{x_{+}} x^{2} \frac{\partial p}{\partial x} \mathrm{~d} x
\end{gathered}
$$

where we recall from (3.6) that $p\left(x_{ \pm}\right)=-2 \bar{\gamma} /\left(h_{0}+\alpha x_{ \pm}\right)$. Performing the integrals in (3.13) and (3.14) using (3.12) gives equations of the form

$$
\begin{aligned}
& f_{\alpha}\left(\alpha, x_{+}, x_{-}\right) \dot{\alpha}+f_{c}\left(\alpha, x_{+}, x_{-}\right) c=f\left(\alpha, x_{+}, x_{-}\right) \\
& g_{\alpha}\left(\alpha, x_{+}, x_{-}\right) \dot{\alpha}+g_{c}\left(\alpha, x_{+}, x_{-}\right) c=g\left(\alpha, x_{+}, x_{-}\right)
\end{aligned}
$$

where the functions $f_{\alpha}, g_{\alpha}$ etc. are determined by performing the integration.

Similarly to the no tilting case, in writing down the torque balance (3.14) we have neglected the rotational inertia of the plate, $I \ddot{\alpha}$, where $I=m \ell^{2} / 3$ is the moment of inertia for a plate of uniform density, width $2 \ell$ and mass per unit length $m$. To understand when this neglect of rotational inertia is appropriate, we note that a droplet centred on the pivot will induce a torque on the plate because the pressure at its menisci becomes unequal immediately following a rotation $\alpha$. The magnitude of this capillary torque, $\tau_{\text {cap }} \propto \Delta p x_{0}^{2}$ where the pressure difference $\Delta p \propto \bar{\gamma} \alpha x_{0} / h_{0}^{2}$ (Bradley et al., 2019); we therefore have that $\tau_{\text {cap }} \propto \bar{\gamma} \alpha x_{0}^{3} / h_{0}^{2}$ dominates the rotational inertia for times $t \gg\left[m h_{0}^{2} \ell^{2} /\left(\bar{\gamma} x_{0}^{3}\right)\right]^{1 / 2}$. This holds in particular for times on the capillary time scale $t_{*}$ provided that $W / \bar{\gamma} \gg\left[m^{2} \bar{\gamma}^{2} \ell^{4} /\left(\mu^{4} V^{3}\right)\right]^{1 / 11}$ or, if $W=m g$, then when $W / \bar{\gamma} \gg\left[\bar{\gamma}^{4} \ell^{4} /\left(\mu^{4} g^{2} V^{3}\right)\right]^{1 / 9}$. Again, this is satisfied for sufficiently large loads (or droplet aspect ratios). 
Finally, since the droplet is free to migrate, we must consider how its menisci move. To conserve droplet volume, we assume that the menisci move with the speed given by the local fluid flux, i.e.

$$
\dot{x}_{ \pm}=-\left.\frac{\left(h_{0}+\alpha x_{ \pm}\right)^{2}}{12 \mu} \frac{\partial p}{\partial x}\right|_{x_{ \pm}} .
$$

This choice ensures that global mass conservation is inherited from local mass conservation (see Taroni \& Vella, 2012, for example).

Consider a small perturbation in angle and droplet position from the equilibrium: $x_{ \pm}= \pm x_{0}+\delta x_{ \pm}$ with $\delta x_{ \pm} \ll x_{0}$ and $\alpha \ll 1$. Eliminating $c$ from the linearizations of (3.15)-(3.17) gives the system of linear equations

$$
\dot{\mathbf{y}}=\frac{1}{t_{*}}\left(\begin{array}{cc}
\frac{5}{2} & -15 \times \frac{h_{0}}{2 x_{0}} \\
-\frac{1}{2} \times \frac{2 x_{0}}{h_{0}} & \frac{5}{2}
\end{array}\right) \mathbf{y} \quad \text { where } \mathbf{y}=\left(\begin{array}{c}
\alpha \\
\frac{\delta x_{+}+\delta x_{-}}{2 x_{0}}
\end{array}\right) .
$$

Note that there are only two linear equations here, after eliminating $c$ from four equations - volume conservation enforces that the droplet width, $x_{+}-x_{-}$, remains constant to linear order in the small perturbations and so we omit this from the linear system of equations.

This matrix has eigenvalues $\sigma=(5 \pm \sqrt{30}) / 2$ with corresponding eigenvectors $\mathbf{y}=\left(\sqrt{30} h_{0}, \mp 2 x_{0}\right)$. We denote the larger of these eigenvalues by $\sigma_{\circlearrowleft}=(5+\sqrt{30}) / 2$, which corresponds to the plate tilting and the droplet moving towards the narrower end; this is the motion that will generally be seen for small perturbations. (While we use an anti-clockwise arrow here, the sign of the rotation is determined by that of the initial perturbation.)

We note that the growth rate of the tilting perturbation, $\sigma_{\circlearrowleft}=(5+\sqrt{30}) / 2$ is larger than the growth rate of the separating perturbation, $\sigma_{\downarrow}=1$ (by choice of the time scale $t_{*}$ ).

\section{Full system: separating and tilting}

Having seen how the modes of plate separation and plate tilting behave in isolation, we now combine these two modes and the effect of shear to obtain a system describing a mobile plate under the action of an applied load supported by the capillary force of a droplet. We seek to understand how the tilting and separating modes interact.

\subsection{Theoretical formulation}

Our full model uses the same theory presented in $\S 3$ : we use Reynolds' equation to describe the fluid flow with the width of the gap described by $h(t)+\alpha(t) x$ (again assuming small angles, $\alpha \ll 1$ ). The resulting pressure gradient within the droplet is (3.5), which contains four unknowns: $\alpha(t), h(t), u(t)$ and $c(t)$. These unknowns are related through the constraints of vertical force balance, (3.8), a known pressure drop, (3.13) and torque balance (3.14). However, since these constraints also involve the a priori unknown meniscus positions, $x_{ \pm}$, the meniscus positions must also be solved for from conservation of mass while including the effect of shear: (3.17) is modified to become

$$
\dot{x}_{ \pm}=-\left.\frac{\left(h+\alpha x_{ \pm}\right)^{2}}{12 \mu} \frac{\partial p}{\partial x}\right|_{x_{ \pm}}+\frac{u}{2} .
$$

To complete this system of equations, we require one more relation, namely a horizontal force balance on the plate. The horizontal forces acting on the plate are an aspect ratio smaller in size than 
the normal forces (see eqn 4.8.18 of Batchelor, 1967, for example), and so it is relatively easy to slide the plate sideways; with no active forcing in this direction, it is not clear a priori whether this horizontal motion will be significant compared to the vertical motion. There are two mechanisms through which the fluid provides a sideways force on the plate: viscous shear as the fluid drags on the plate as it flows past, and the pressure acting normally on the plate having a horizontal component because of the plate's slope. Summing these contributions, the horizontal force balance is found to be

$$
0=\int_{x_{-}}^{x_{+}}-\frac{h+\alpha x}{2} \frac{\partial p}{\partial x}+\frac{\mu u}{h+\alpha x}-\alpha p \mathrm{~d} x=\int_{x_{-}}^{x_{+}}-\frac{\alpha}{2} p+\frac{\mu u}{h+\alpha x} \mathrm{~d} x
$$

where we have integrated the pressure gradient term by parts.

Note that we have ignored the plate inertia in the horizontal direction, $m \dot{u}$, when taking a horizontal force balance, (4.2), despite the shear forces on the plate being an aspect ratio smaller in size than the normal forces. Using a typical horizontal velocity scale $x_{0} / t$, we find that this inertia is negligible for times $t \gg\left[m x_{0} / \bar{\gamma}\right]^{1 / 2}$; for times on the capillary timescale $t_{*}$, this holds when the load is sufficiently large: $W / \bar{\gamma} \gg\left[m^{2} \bar{\gamma}^{2} /\left(\mu^{4} V\right)\right]^{1 / 5}$. Note that if the load is the weight of the plate, $W=m g$, then this becomes $W / \bar{\gamma} \gg\left[\bar{\gamma}^{4} /\left(\mu^{4} g^{2} V\right)\right]^{1 / 3}$.

The integral condition (4.2) can be evaluated, using the vertical force balance (3.8), to give a solution for the shear velocity $u(t)$ in terms of the plate and droplet positions

$$
u=\frac{-W \alpha^{2}}{2 \mu[\log (h+\alpha x)]_{\mathrm{drop}}} .
$$

We find that, to balance the horizontal forces on the plate, the substrate must shear to the left when the plate is tilted so that $\alpha$ is positive (in the sense of fig. 3); in the lab frame, where the substrate is fixed, the plate moves to the right as the capillary pressure pulls on the angled plate, with the droplet moving towards the left end of the plate.

\subsection{Non-dimensionalization}

For simplicity we non-dimensionalize our problem using the equilibrium separation $h_{0}$, the associated droplet width $2 x_{0}$, and the time scale $t_{*}$ from (3.10); in other words, we define the new variables

$$
H=\frac{h}{h_{0}}, \quad X=\frac{x}{2 x_{0}}, \quad \beta=\frac{2 x_{0}}{h_{0}} \alpha, \quad P=\frac{p}{2 \bar{\gamma} / h_{0}}, \quad T=\frac{t}{t_{*}}, \quad U=\frac{u}{2 x_{0} / t_{*}} .
$$

In these dimensionless variables, the unstable equilibrium solution is now $H=1, X_{ \pm}= \pm 1 / 2$ with $\beta=0$, and the droplet has unit volume. The new rescaled angle, $\beta$, may be thought of as the dimensionless height difference over the droplet's initial width that is caused by tilting. We note that, since the pressure is rescaled by a factor $\cos \theta$ (recalling $\bar{\gamma}=\gamma \cos \theta$ ), this analysis is not valid as $\theta \rightarrow \pi / 2$. This is consistent with our neglect of the tension force applied at the edges $(\propto \gamma \sin \theta)$ in comparison to the force generated by the capillary suction within the droplet $\left(\propto x_{0} \times \gamma \cos \theta / h_{0}\right)$, which requires $\tan \theta \ll x_{0} / h_{0}$.

The dimensionless lubrication pressure gradient is

$$
\frac{\partial P}{\partial X}=12 \frac{\dot{\beta} X^{2}+2 \dot{H} X+C}{(H+\beta X)^{3}}+\frac{12 U}{(H+\beta X)^{2}}
$$

where $C=C(T)$ is an (as yet unknown) function of time $T$ and dots now denote differentiation with respect to $T$. 
Since the pressure at each end is constrained by the capillary pressure $P\left(X_{ \pm}\right)=-1 /\left(H+\beta X_{ \pm}\right)$we have

$$
\int_{X_{-}}^{X_{+}} \frac{\partial P}{\partial X} \mathrm{~d} X=[P]_{\mathrm{drop}}=-\left[(H+\beta X)^{-1}\right]_{\mathrm{drop}},
$$

which is the dimensionless version of (3.13). Furthermore, we impose vertical force and torque balances on the loaded plate, (3.8) and (3.14), which can be written in dimensionless terms as

$$
\begin{aligned}
& 1=\int_{X_{-}}^{X_{+}}-P \mathrm{~d} X=-[X P]_{\mathrm{drop}}+\int_{X_{-}}^{X_{+}} X \frac{\partial P}{\partial X} \mathrm{~d} X \\
& 0=\int_{X_{-}}^{X_{+}} X P \mathrm{~d} X=\frac{1}{2}\left[X^{2} P\right]_{\mathrm{drop}}-\frac{1}{2} \int_{X_{-}}^{X_{+}} X^{2} \frac{\partial P}{\partial X} \mathrm{~d} X,
\end{aligned}
$$

respectively. Surprisingly, this non-dimensionalization does not leave any further parameter in the problem - the load $W$ might be expected to feature, but in effect disappears because the equilibrium gap width and droplet width ( $h_{0}$ and $2 x_{0}$, respectively) are used to non-dimensionalize the vertical and horizontal length scales separately, whilst being related through the equilibrium condition (3.3). As a result, the capillary pressure scale $p^{*}=2 \bar{\gamma} / h_{0}$ can also be written $p^{*}=W /\left(2 x_{0}\right)$, and the term representing the applied vertical load becomes unity. form

We note that, after substituting (4.5) and integrating, the equations (4.6)-(4.8) can be written in the

$$
\begin{aligned}
& 12 I_{2} \dot{\beta}+24 I_{1} \dot{H}+12 I_{0} C+12\left(H I_{0}+\beta I_{1}\right) U=-\left[\frac{1}{H+\beta X}\right]_{\text {drop }} \\
& 12 I_{3} \dot{\beta}+24 I_{2} \dot{H}+12 I_{1} C+12\left(H I_{1}+\beta I_{2}\right) U=1-\left[\frac{X}{H+\beta X}\right]_{\text {drop }} \\
& 12 I_{4} \dot{\beta}+24 I_{3} \dot{H}+12 I_{2} C+12\left(H I_{2}+\beta I_{3}\right) U=-\left[\frac{X^{2}}{H+\beta X}\right]_{\text {drop }}
\end{aligned}
$$

respectively, where the integrals $I_{n}=I_{n}\left(H, \beta, X_{+}, X_{-}\right)$are defined by

$$
I_{n}=\int_{X_{-}}^{X_{+}} \frac{X^{n}}{(H+\beta X)^{3}} \mathrm{~d} X
$$

The edges of the droplet move due to the fluxes of fluid there (3.17), which in dimensionless form becomes

$$
\dot{X}_{ \pm}=-\left.\frac{\left(H+\beta X_{ \pm}\right)^{2}}{24} \frac{\partial P}{\partial X}\right|_{X_{ \pm}}+\frac{U}{2}=-\frac{\dot{\beta} X_{ \pm}^{2}+2 \dot{H} X_{ \pm}+C}{2\left(H+\beta X_{ \pm}\right)} .
$$

From the horizontal force balance, (4.2), we find the dimensionless shear velocity, $U(T)$, is

$$
U=\frac{-\beta^{2}}{4[\log (H+\beta X)]_{\mathrm{drop}}} .
$$

Once we have substituted for the value of $U$, the system (4.9)-(4.11) with (4.13) gives five equations involving $\beta(T), H(T), X_{ \pm}(T)$ and $C(T)$ and the derivatives of $\beta, H \& X_{ \pm}$, i.e. a system of differentialalgebraic equations. Eliminating $C$ leads to a fourth order system of ODEs, which is given in Appendix A for completeness. 

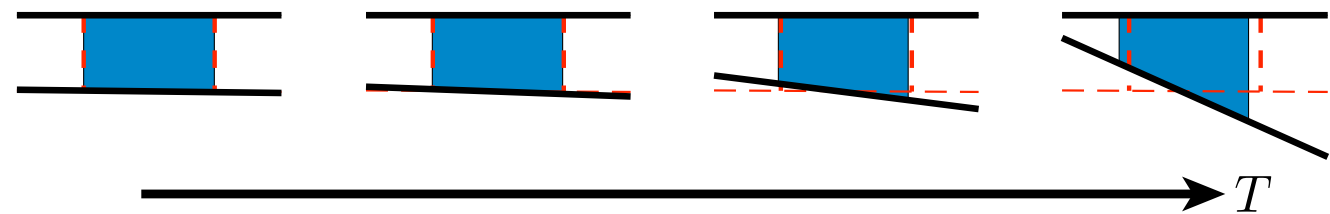

FIG. 6. Snapshots generated from the numerical solution at early times showing the initial tilting of the plate when $\delta H(0)=$ $\beta(0)=10^{-2}$ and the droplet begins centrally, $X_{+}(0)+X_{-}(0)=0$. The substrate is at the top of each plot, while the lower plate is free to rotate and separate. The snapshots are presented in the frame of reference of the plate (the substrate shears to the left). Solutions are evaluated at dimensionless times $T=0.2,0.4,0.6,0.8$ with the initial position overlaid as red dashed lines.

\subsection{Numerical solutions}

This dimensionless system of ODEs has no parameters, and is solved numerically from given initial conditions using MATLAB's stiff ODE solver ode15s.

In fig. 6, we show an example of the evolution of the system at various early times when starting with a small perturbation from the unstable equilibrium solution in both the tilt and separation, namely $\delta H(0)=\beta(0)=10^{-2}$. From this solution, it would appear that the predominant motion of the plate is to tilt rather than separate. This raises the question of why this is what is seen at early times - a question that we turn to investigate using linear stability analysis.

\subsection{Linear stability analysis}

Considering small perturbations about the equilibrium solution $H=1+\delta H, X_{ \pm}= \pm 1 / 2+\delta X_{ \pm}$, with $\delta H, \delta X_{ \pm}, \beta, C, U \ll 1$, we find that (ignoring higher order terms) the linearized pressure gradient is

$$
\frac{\partial P}{\partial X}=12 \dot{\beta} X^{2}+24 \dot{\delta H} X+12 C+12 U .
$$

Substituting this into (4.6)-(4.8) and (4.13) gives the linear system of equations

$$
\begin{aligned}
\dot{\beta} & =\beta-12(C+U) \\
2 \dot{\delta} H & =\delta H-\left(\delta X_{+}-\delta X_{-}\right) \\
\frac{3}{10} \dot{\beta} & =\frac{1}{2} \beta-2(C+U)-2\left(\delta X_{+}+\delta X_{-}\right) \\
\delta \dot{X}_{+} & +\delta \dot{X}_{-}+\frac{1}{4} \dot{\beta}=-C \\
\delta \dot{X}_{+} & -\delta \dot{X}_{-}+\dot{\delta} H=0 .
\end{aligned}
$$

Note that volume conservation

$$
1=\left(X_{+}-X_{-}\right)\left[H+\frac{\beta}{2}\left(X_{+}+X_{-}\right)\right]
$$

becomes $\delta X_{+}-\delta X_{-}+\delta H=0$ upon linearization, and so the last equation in (4.16) is simply the time derivative of the linearized volume conservation. This means that the order of the linearised system can be reduced by 1: $\delta X_{+}-\delta X_{-}$can be replaced in favour of $\delta H$, and we can then consider the evolution of 
droplet position using $\delta X_{+}+\delta X_{-}$and $\delta H-$ the individual values of $\delta X_{+}$and $\delta X_{-}$can be reconstructed if desired from volume conservation.

Eliminating $C$ and $\delta X_{+}-\delta X_{-}$from (4.16), and rearranging, we find that the system simplifies to

$$
\dot{\mathbf{Y}}=\left(\begin{array}{ccc}
1 & 0 & 0 \\
0 & \frac{5}{2} & -15 \\
0 & -\frac{1}{2} & \frac{5}{2}
\end{array}\right) \mathbf{Y}+\left(\begin{array}{l}
0 \\
0 \\
U
\end{array}\right)
$$

where $\mathbf{Y}=\left(\delta H, \beta, \delta X_{+}+\delta X_{-}\right)$.

If the system is constrained so that the plate cannot migrate horizontally, $U=0$, the linear system decouples into precisely the separating and tilting modes that were discussed in $\S 3$ and are illustrated in fig. 4. One mode has eigenvalue $\sigma_{\uparrow}=1$ and corresponds to the load falling or rising with no tilting. The fastest growing mode is $\sigma_{\circlearrowleft} \approx 5.24$, which involves the plate tilting and the droplet migrating towards the narrower end.

For an unconstrained free plate, we must also include the effect of shearing and so consider the horizontal force balance on the plate, (4.2). Linearizing the dimensionless shear speed $U$ in (4.14), we find

$$
U=-\frac{\beta}{4}
$$

This modifies the evolution of the plate so that now

$$
\dot{\mathbf{Y}}=\left(\begin{array}{ccc}
1 & 0 & 0 \\
0 & \frac{5}{2} & -15 \\
0 & -\frac{3}{4} & \frac{5}{2}
\end{array}\right) \mathbf{Y}
$$

The motion again decouples into the two separate modes shown in fig. 4. However, the tilt-and-migrate mode now has eigenvalues $\sigma_{ \pm}=(5 \pm 3 \sqrt{5}) / 2$, and the linearized system has solutions of the form

$$
\begin{aligned}
\delta H(T) & =\delta H(0) e^{T} \\
\beta(T) & =A e^{\sigma_{+} T}+B e^{\sigma_{-} T} \\
\delta X_{+}(T)+\delta X_{-}(T) & =\frac{1}{2 \sqrt{5}}\left(-A e^{\sigma_{+} T}+B e^{\sigma_{-} T}\right)
\end{aligned}
$$

Including the shear has the effect of amplifying the growth rate of the tilting mode, which now grows with $\sigma_{+} \approx 5.85>\sigma_{\circlearrowleft}$.

In fig. 7, we plot the numerical solution to the full equations at early times compared to the predictions of the linear stability analysis for different perturbation sizes. Here, we pick initial conditions such that the $\sigma_{-}$mode is not present, i.e. we choose $\beta(0)=-2 \sqrt{5}\left[\delta X_{+}(0)+\delta X_{-}(0)\right]$ so that the coefficient $B$ vanishes, $B=0$ in (4.21). We see that when the perturbations are both small and of similar size, the evolution agrees well with the linear stability analysis. However, when the angular perturbation is significantly larger than the height perturbation then the evolution of $\delta H$ deviates from the expected value much sooner - the tilt and migrate mode dominates and affects the evolution of the separation.

Fig. 8 shows the evolution of the height, angle and droplet centre's position when the droplet centre is initially aligned with the centre of mass of the plate, $X_{-}(0)=-X_{+}(0)$ (i.e. we are no longer forcing the system into the solely growing mode, since $B \neq 0$ ). For this case the numerical solutions also follow 
(a)

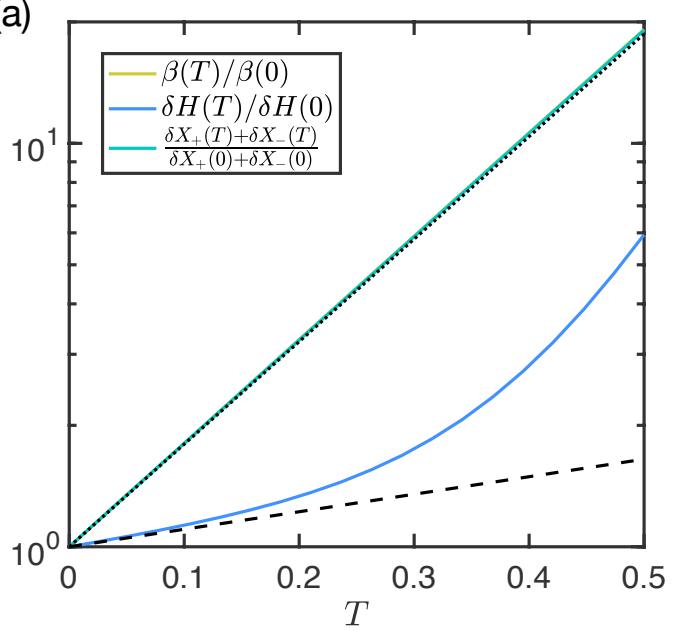

(b)

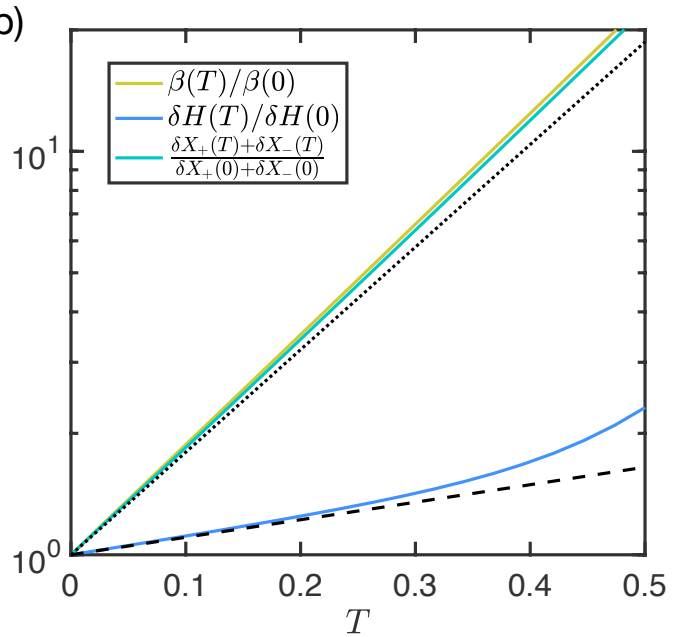

FIG. 7. The evolution of the perturbations to the height, angle and droplet position at early times for small perturbations to the equilibrium solution, compared to the predictions of a linear stability analysis (dashed line for height, dotted line for angle and droplet centre). Here the initial perturbation is chosen to excite just the growing mode, $B=0$ in (4.21). Each plot has an initial angular perturbation of $\beta(0)=10^{-2}$, with the value of the initial height perturbation varied: (a) $\delta H(0)=10^{-3}$ and (b) $\delta H(0)=10^{-2}$.

the predictions of the linear stability analysis well at early times: the influence of the constant $B \neq 0$ decays quickly so that the numerical results are well approximated by the later time behaviour of the linear stability analysis, i.e. $\beta \approx A \exp \left(\sigma_{+} t\right)$. This agreement for a generic initial condition further suggests that the linear stability analysis is a good predictor of the initial motion and emphasizes the dominance of the tilt-and-migrate mode in the plate dynamics. For simplicity, from here onwards the calculations shown will be for an initially centred droplet, as in this figure.

\section{Does the load fall or stick?}

We have seen that a linear stability analysis suggests that there can be two distinct initial motions of the plate for small perturbations, depending on the relative sizes of perturbations to the separation and angle of the plate. However, this does not tell us the response of the system at later times: is the loaded plate ultimately pulled in to stick to the fixed substrate indefinitely, or does it instead detach? We turn to investigate this now, using the numerical solutions of our system (4.9)-(4.13).

If there is little or no tilt, then we expect that the motion will largely follow that considered by Ward (2011), with detachment occurring for any outward perturbations and adhesion maintained if the load is perturbed inwards.

However, based on the analysis we have seen so far, any tilt that is initially present in the system grows more rapidly than a comparable perturbation to the separation of the centre. This rotation would lead to contact between the rigid substrate and the load at one edge. In this case, the ultimate fate of the attached load is expected to depend a great deal on when this contact occurs, and what happens beyond it. In addition to how the plate is initially perturbed, the plate half-width $\ell$ will be important in determining these outcomes. We turn to consider the effect of this contacting scenario now. 
(a)

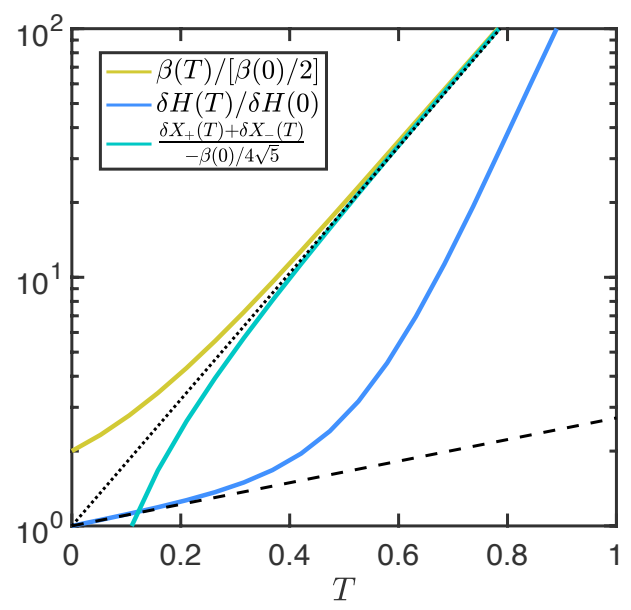

(b)

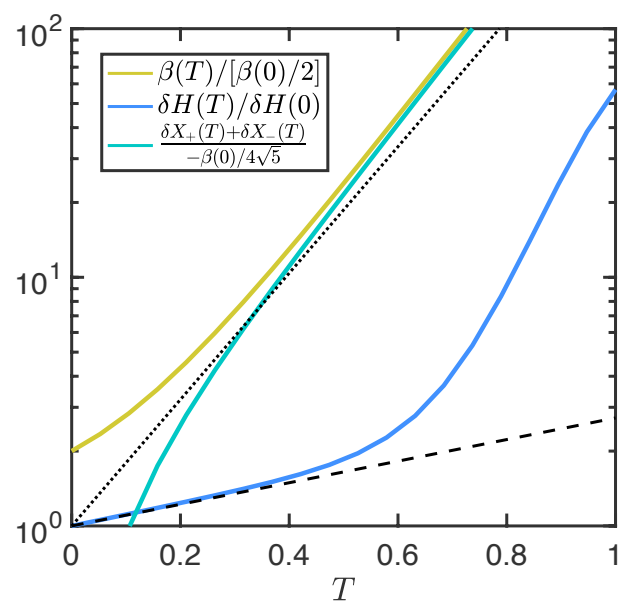

FIG. 8. Comparison between the prediction of the linear stability analysis (dashed and dotted lines) and numerical solutions of the full problem (coloured curves). Here, the parameter values are precisely as in fig. 7, but the droplet starts at the centre of the plate, $X_{-}(0)=-X_{+}(0)$, thereby also exciting the exponentially decaying mode, $\sigma_{-}, B \neq 0$ in (4.21). Note that the angle and droplet-centre position have been normalised by the pre-factor for the growing mode, $A=\beta(0) / 2$, so that perfect agreement with the unstable mode of the linear stability analysis at late times would be $\exp \left(\sigma_{+} t\right)$ with $\sigma_{+} \approx 5.85$, which is shown by the dotted line. The dashed line shows the corresponding prediction of linear stability analysis for $H(T)$.

\subsection{Corner contact case}

If contact occurs then the physics determining the motion are modified due to the presence of a reaction force at the corner. This means that it is no longer appropriate to consider a vertical force balance on the load as a whole (which would simply determine the size of the reaction force) and, further, that it is more natural to consider a torque balance about the contact point. In addition, we will assume that there is sufficient friction between the plate and substrate that the point of contact remains fixed; there is no longer any shearing of the plate relative to the substrate, $U=0$, and we may also omit the horizontal force balance. For simplicity, we focus on the case where contact occurs at the left edge, $X=-L$ for a dimensionless plate half-width $L=\ell /\left(2 x_{0}\right)$; the same equations hold at the right edge once the change $L \rightarrow-L$ is made.

Once contact has occurred, the wedge angle and separation at the centre are coupled through the geometric condition $H=\beta L$; as a result, the problem remains well-defined, despite the loss of vertical force balance. Using this simplification, we then find that the lubrication pressure gradient (4.5) is

$$
\frac{\partial P}{\partial X}=\frac{12}{\beta^{3}} \frac{\left(X^{2}+2 L X\right) \dot{\beta}+C}{(L+X)^{3}}=\frac{12}{\beta^{3}} \frac{(L+X)^{2} \dot{\beta}+D}{(L+X)^{3}}
$$

where we have defined a new function of time $D(T)=C(T)-L^{2} \dot{\beta}(T)$ to simplify the calculations here. Integrating over the width of the droplet, gives us a condition on the pressure equivalent to (4.6)

$$
\frac{12}{\beta^{3}}\left(J_{-1} \dot{\beta}+J_{-3} D\right)=-\frac{\left[(L+X)^{-1}\right]_{\mathrm{drop}}}{\beta}
$$

where $J_{n}\left(X_{+}, X_{-} ; L\right)=\int_{X_{-}}^{X_{+}}(L+X)^{n} \mathrm{~d} X$. 
The torque balance (taken about the contacting corner for simplicity) can be written in dimensionless terms as

$$
L=\int_{X_{-}}^{X_{+}}-(L+X) P \mathrm{~d} X=\left[-\frac{(L+X)^{2}}{2} P\right]_{\mathrm{drop}}+\int_{X_{-}}^{X_{+}} \frac{(L+X)^{2}}{2} \frac{\partial P}{\partial X} \mathrm{~d} X
$$

which can alternatively be written as

$$
\frac{6}{\beta^{3}}\left(J_{1} \dot{\beta}+J_{-1} D\right)=L-\frac{[L+X]_{\text {drop }}}{2 \beta}
$$

and the droplet edges move due to (4.13), which is now

$$
\dot{X}_{ \pm}=-\frac{\left(L+X_{ \pm}\right)^{2} \dot{\beta}+D}{2 \beta\left(L+X_{ \pm}\right)} .
$$

We solve the differential-algebraic equations (5.2), (5.4) and (5.5) to find the plate and droplet evolution by first eliminating $D$ to get a system of ODEs; the full system is detailed in Appendix A.

\subsection{Stick or fall}

We simulate the motion of the loaded plate for a variety of different plate half-widths $L$ and perturbations from equilibrium. Details of the conditions used are given in Appendix B. If the corner of the plate contacts the substrate then we allow the motion to continue but switch to using the torque balance appropriate to the corner contact case, i.e. (5.3).

In some of these contacting cases, we find that the droplet migrates far enough to reach the edge of the plate (now the corner of the wedge); an asymptotic analysis shows that this occurs in finite time - see Appendix C. Once the fluid is in the corner, there is no fluid flux there and so we can solve the remainder of the motion analytically: assuming the droplet reaches the left-most corner, a torque balance (5.3) gives the evolution of the angle

$$
L=\frac{X_{+}+L}{2 \beta}+\frac{3\left(X_{+}+L\right)^{2}}{\beta^{3}} \dot{\beta} .
$$

Note that this is simply (5.4) with $D=0$ and $X_{-}=-L$. The sign of $\dot{\beta}$ is determined by whether the capillary torque $\left(X_{+}+L\right) / 2 \beta$ is greater or less than the load torque $L$ : if

$$
X_{+}+L-2 L \beta>0
$$

at the instant the droplet meniscus reaches the corner, then $\dot{\beta}<0$ and $\beta$ decreases with increasing time. Decreasing $\beta$ ensures that the inequality in (5.7) holds for all subsequent times if it holds initially, and so we conclude that the plate ultimately sticks if the inequality (5.7) holds at the instant that the left hand meniscus reaches the corner, $X_{-}=-L$. (A similar condition holds if the droplet first reaches the right hand edge, albeit with $L$ replaced by $-L$ and $X_{+}$replaced by $X_{-}$, since $\beta<0$ in this case.)

Since there is no material parameter remaining in the problem after the non-dimensionalization, we expect that the fate of a particular load will be determined by the plate half-width, $L$, and the initial conditions. To explore this relationship, we fix the initial angle $\beta(0)$ and vary the separation perturbation $\delta H(0)$ and plate half-width $L$. Fig. 9 shows regime diagrams of the regions of the $[L, \delta H(0)]$ parameter 

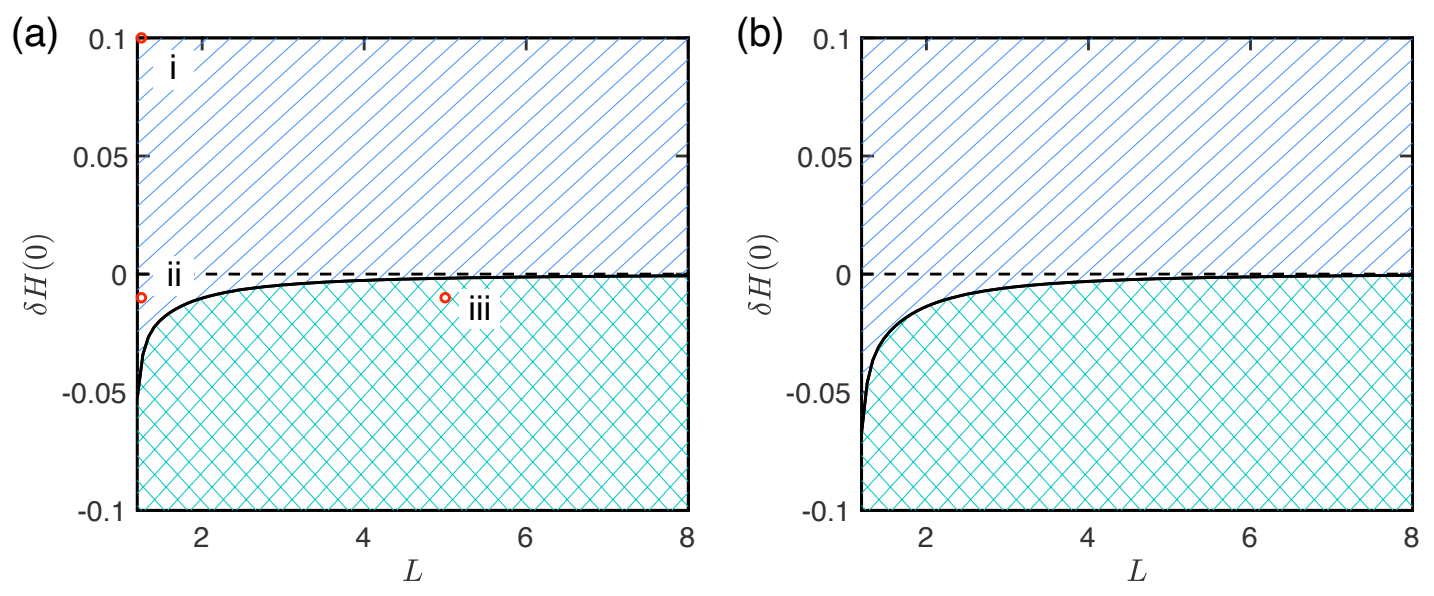

FIG. 9. Plot showing results of whether the load ultimately adheres to the substrate (cross hatched region) or ultimately detaches (striped region) for different values of the plate half-width $L$ and perturbation size $\delta H(0)$ when the droplet begins at the plate centre, $X_{+}(0)+X_{-}(0)=0$. Results are shown with initial angular perturbations: (a) $\beta(0)=10^{-2}$, and (b) $\beta(0)=10^{-1}$. The horizontal dashed line indicates no initial separation perturbation, $\delta H(0)=0$; linear stability analysis with a parallel plate predicts that above this line the plate detaches, while below it adhesion persists. The red circles in (a) denote the parameter values for the snapshots presented in fig. 10.

space for which the plate ultimately sticks or de-adheres when $\beta(0)=10^{-2}, 10^{-1}$. (Here, the plotted boundary was calculated using interval bisection.)

Qualitatively, we find that when the plate half-width $L$ is smaller, the load detaches even for some inward perturbations, $\delta H(0)<0$. At larger load sizes, this effect is reduced, and the boundary between the load sticking or detaching is much closer to what would be expected from the parallel plate's linear stability analysis in $\S 3$; specifically, the boundary between sticking and detaching is very close to $\delta H(0)=0$. Additionally (comparing fig. $9 \mathrm{~b}$ to fig. $9 \mathrm{a}$ ) we note that when the angle perturbation is larger, a greater inward perturbation is needed to stick at smaller $L$. These results show that the presence of even small amounts of rotation can fundamentally change the ultimate fate of a load adhered via surface tension.

Looking in more detail at the evolution of the numerical solutions in these different regions (see, for example, fig. 10) we find that the rotation of the plate results in corner contact on relatively short timescales. Once this has occurred, the subsequent evolution is determined by the competition between load and capillary torques about the corner. In a few cases we find that some time after contact the droplet reaches the corner and we must apply (5.7).

Smaller-sized plates have a relatively large angle and the capillary torque may be beaten by the load torque, resulting in detachment, even for some inward perturbations (see fig. 10ii). Plots of the plate midpoint separation $H(T)$ with time for a plate of half-width $L=1.25$ are shown in fig. 11a. In particular, we show two cases where the initial perturbation was inwards and yet the height increases and plate separates, qualitatively similar to the experimental trajectories of anomalous detachment highlighted in fig 5 a.

For longer plates, contact at the corner occurs sooner and the plate angle is smaller. For some perturbations where shorter plates would detach, the capillary torque levers the plate upwards (see fig. 10iii). In fig. $11 \mathrm{~b}$, we see the midpoint separation $H(T)$ for a plate of half-width $L=5$. The initial contact 

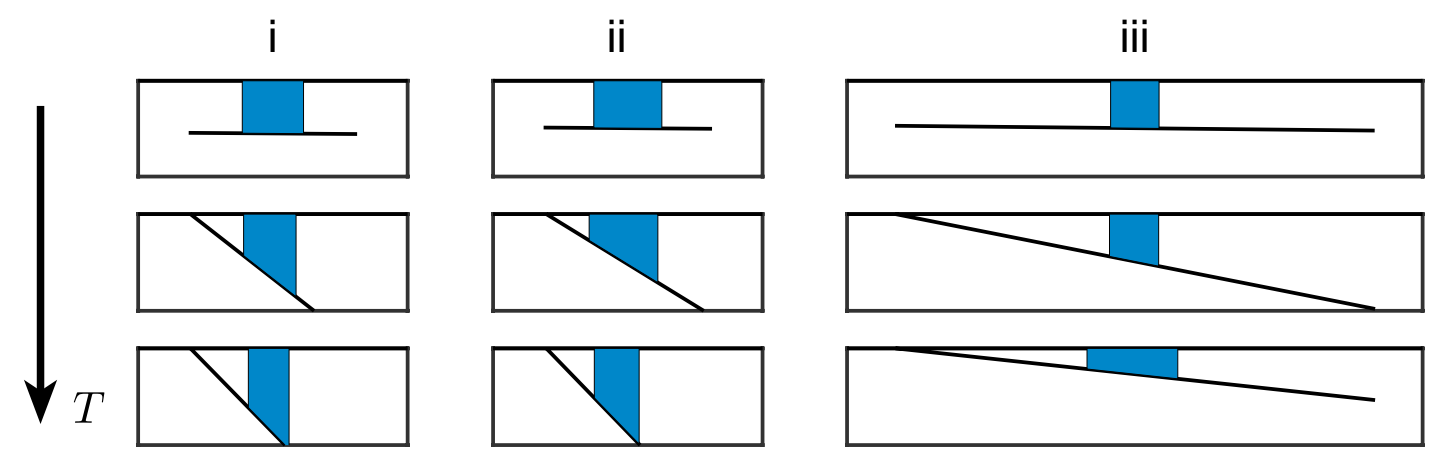

FIG. 10. Plots of plate and droplet positions at different time intervals, showing their evolution in the lab frame, as well as the role of plate size. The initial conditions used and times plotted are: (i) $\delta H(0)=+10^{-1}, L=1.25$, at times $T=0,0.5,0.65$, (ii) $\delta H(0)=+10^{-2}, L=1.25$, at times $T=0,1,2.75$, (iii) $\delta H(0)=+10^{-2}, L=5$, at times $T=0,1,10$. In all cases $\beta(0)=10^{-2}$. Note that the evolution of the mean separation, $H(T)$, in each case is shown in fig. 11.

occurs earlier than in fig. 11a (the plate is longer and so does not need to tilt as much) and the perturbation $\delta H(0)=-10^{-2}$ does not anomalously detach for this larger plate case, even though it does when $L=1.25$. Moreover, the overall stick/detach behaviour is similar to what would be expected from the parallel plate's linear stability (compare to fig. $5 b$ ) - for longer plates, the tilting does not appear to have a significant effect on whether the loaded plate sticks or detaches.

Our linear stability analysis showed that the shearing of the fluid, and hence horizontal migration of the plate in the lab frame, amplifies the initial growth rate of the tilting motion. It is natural, therefore, to wonder whether the total horizontal migration (prior to contact) is significant in comparison to the plate length. In fig. 12a, we show the horizontal motion of a plate in the lab frame from its initial position as a function of time, $X_{\text {plate }}(T)=-\int_{0}^{T} U(\tilde{T}) \mathrm{d} \tilde{T}$, for a plate of half-width $L=1.25$ when subject to different initial vertical perturbations at the centre of mass, $\delta H(0)$. The migration of the plates speeds up over time, but contact with the substrate is made on relatively short timescales; hence the horizontal motion is limited to a small fraction of the plate length (the horizontal motion of the plate ends is indiscernible between the snapshots of fig. 10). For longer plates, the same trajectories are followed but the plates contact the substrate sooner, and so the horizontal deviation of the plate is reduced even further. In fig. $12 \mathrm{~b}$, we show the total distance moved by the plate up to the point of contact as a function of the plate half-width, $L$. The overall sideways drift decreases with increasing plate width, appearing to follow a scaling $X_{\text {plate }} \sim L^{-1}$; this can be rationalized by remembering that at early times the plate moves at a speed $U \propto \beta$ and the angle grows exponentially until it has reached an angle of size $1 / L$. In conclusion, the plate's sideways drift is small (in comparison to the plate length), despite having a noticeable effect on the growth rate of instability.

Finally, we shall address the expected final state of the plate and droplet in our system. Our theory is a lubrication model - assuming a thin film of fluid — and so is not able to account for rupture of the bridge as the plate separation increases; in the situations where we have concluded that the plate 'detaches' or 'falls', we expect the fluid to eventually rupture as the bridge elongates. In the cases where the plate 'sticks' or 'adheres', the droplet will eventually be squeezed between the two surfaces 
(a)

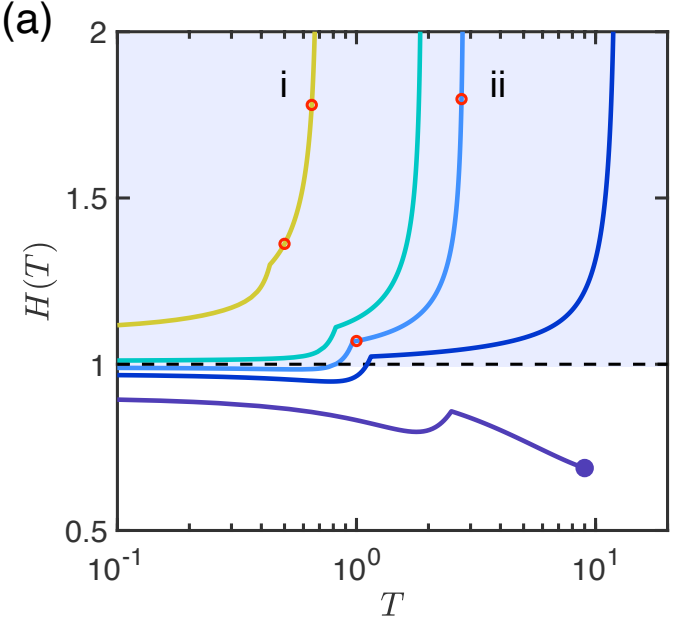

(b)

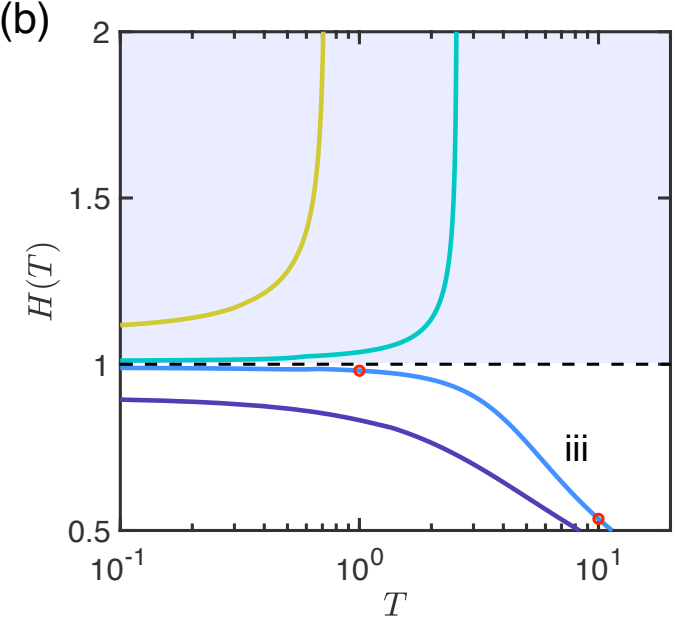

FIG. 11. The evolution of the dimensionless separation of the plate centre of mass, $H(T)$, for various values of the perturbation from equilibrium, $\delta H(0)$, and two different plate half-widths (a) $L=1.25$, and (b) $L=5$. Each plot shows trajectories with initial separation perturbations of $\delta H(0)= \pm 10^{-2}, \pm 10^{-1}$; in (a) an additional trajectory with $\delta H(0)=-0.03$ is also shown. In all cases the angle perturbation is $\beta(0)=10^{-2}$. Filled circles show when the numerical integration terminates due to the droplet nearing the corner (see Appendix B). The three solutions labelled with roman numerals are the trajectories corresponding to the snapshots shown in fig. 10, with the red circles indicating the corresponding time points for plots with $T>0$. The shaded region in each plot is the region in which the usual parallel stability analysis would predict detachment; note that in (a) some trajectories that begin in the lower (adhering) region ultimately detach.

(a)

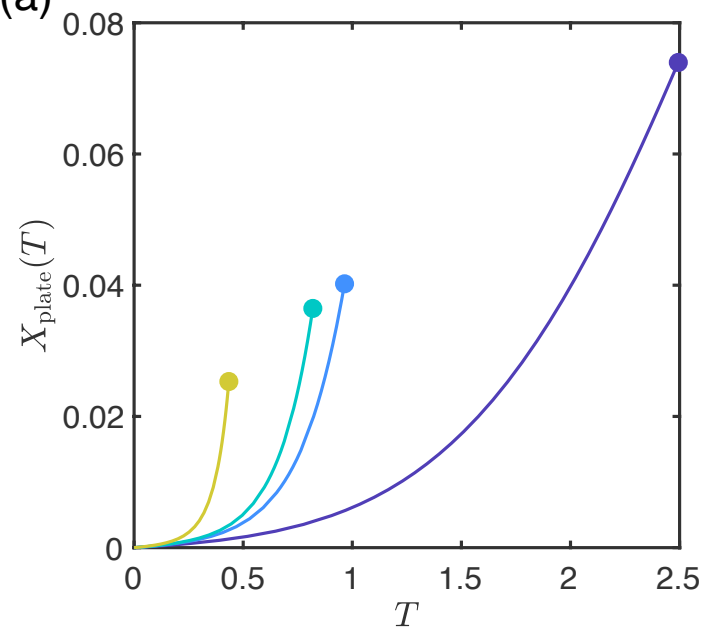

(b)

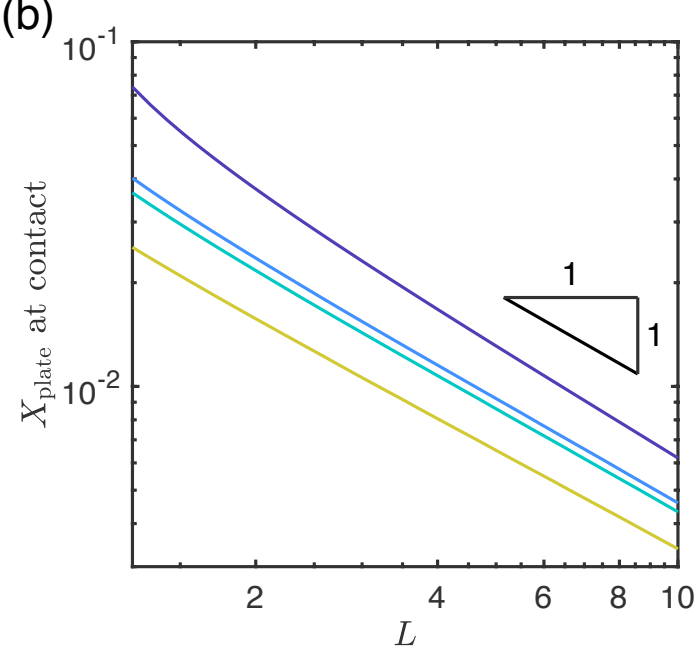

FIG. 12. (a) The horizontal position of the centre of mass of the plate, $X_{\text {plate }}(T)$, in the lab frame as a function of time $T$ for various perturbations $\delta H= \pm 10^{-2}, \pm 10^{-1}$ (colours correspond to those shown in fig. 11a). The angle perturbation is $\beta(0)=10^{-2}$ and the plate has half-width $L=1.25$. The filled circles denote when the plate contacts the substrate at a corner and the shearing motion ends. (b) The total distance the plate has moved before contacting the substrate as a function of the plate length for the same perturbations as in (a). 
sufficiently that the menisci reach both edges of the plate. Once this has occurred, we expect that the plate loses contact with the substrate and the menisci become pinned at the plate edges (the contact angle is no longer fixed). The bridge can then reach a stable equilibrium state close to the substrate when the edges of the droplet are pinned, there is an equilibrium solution that is stable to both constant force and constant displacement perturbations (see Slater et al., 2014, for example).

\section{Conclusions}

In this paper, we have considered the motion of a loaded plate that is joined to a flat substrate by the surface tension of a capillary bridge. Allowing the plate to freely tilt and the droplet to migrate in response to the resulting sloping geometry allows for a variety of different dynamical processes to be explored.

For a given volume of fluid and applied load, we find that there is one equilibrium solution and a linear stability analysis shows that this is unstable to perturbations at fixed load. The linear stability decouples into two distinct modes: (i) motion perpendicular to the substrate (either towards or away) or (ii) tilting of the plate and migration of the droplet into the narrower gap. The tilting mode grows fastest, and its growth is amplified by the sideways shearing of the plate, but which mode is seen will depend on the size of the initial perturbation from equilibrium - if perturbations to the angle $\beta$ and separation $H$ are orders of magnitude different then one may dominate over the other.

Studying the evolution at later times numerically, we determined whether the load is pulled in to stick closely to the substrate or ultimately detaches. In exploring this motion, we had to account for instances in which there was sufficient tilt for the plate to pivot around a corner, as well as what happened when the droplet reaches this corner. The final state of the plate depends sensitively on the plate size (beyond the initial droplet size) as well as the relative sizes of the perturbation. This suggests that it is important to take the tilt into account in such a capillary system. Moreover, it is possible that the presence of even a relatively small amount of this tilting motion may help to explain why the boundary between sticking and detaching was not found at $H=1$ in the experiments of Ward (2011) - these experiments were performed with relatively large droplets (or small plates), so that $L \sim 1$. As such, we expect them to be in the regime where sufficiently small negative perturbations cause detachment, rather than adhesion, just as is observed experimentally (see fig. 5a). It would be interesting to see whether experiments agree with our dependence of adhesion on the plate half-width $L$.

Our model and analysis is limited to a relatively simple two-dimensional system. Nevertheless, it produces some interesting results that may carry over (at least qualitatively) into more realistic threedimensional systems. It would be interesting to see how the added components of an additional direction of curvature, as well as the possibility of fluid flow in other directions and plate twisting, would affect the droplet-plate motion. A key complication here is the evolution of the shape of the droplet's footprint as it migrates into the wedge; experiments by Reyssat (2014) for a droplet migrating into a wedge with fixed angle suggested that the droplet's contact line may remain approximately circular. This previous work also suggests that the difference between two-dimensional and three-dimensional droplets is quantitative, rather than qualitative (Reyssat, 2014; Gorce et al., 2016): in both cases the leading meniscus reaches the corner in finite time and at a constant speed. Nevertheless, whether this qualitative similarity between the 2D and 3D cases, together with the simple contact line shape of the droplet, remain when the wedge angle evolves dynamically, as described here, remains an open question. In particular, the three-dimensional problem would require consideration of the stability of the fluid interface - during detachment, air would displace the more viscous liquid in the droplet, and we should generally expect the interface to be unstable to a Saffman-Taylor instability, as shown in the experiments of Ward (2011). 
A number of other physical effects have been neglected in the analysis that we have presented here. For example, we considered only an undeformable loaded surface while the experiments presented by Butler et al. (2019) involved a flexible membrane subject to a load. Including the deformation of the loaded surface would allow for peeling (as studied by McEwan \& Taylor, 1966, for example) to occur in addition to the tilting and separation considered here - conversely, peeling with a time-dependent peeling angle, as would occur in this case, would provide an interesting extension of the classic McEwan \& Taylor (1966) problem. Contact angle hysteresis may also play an important role in limiting the motion of a fluid droplet in a rigid system, which would likely eventually force the system into the separating mode over tilt and migrate. Indeed, Ataei et al. (2017) found that in the presence of contact angle hysteresis, the tilt must exceed a critical angle for the droplet to begin migrating, which may limit the effect of tilting mentioned here. These extensions would each be worth further work; which of them is most pressing should perhaps be guided by the results of further experiments.

\section{Acknowledgement}

The research leading to these results received funding from EPSRC Grant No. EP/N509711/1 (MB), the Corpus Christi Shand Green-MI Scholarship (MB), the European Research Council under the European Union's Horizon 2020 Program/ERC Grant 637334 (DV) and a Philip Leverhulme Prize (DV).

\section{REFERENCES}

Argentina, M., Skotheim, J. M. \& Mahadevan, L. (2007) Settling and swimming of flexible fluid-lubricated foils. Phys. Rev. Lett., 99(22), 224503.

Ataei, M., Chen, H., Tang, T. \& Amirfazli, A. (2017) Stability of a liquid bridge between nonparallel hydrophilic surfaces. J. Colloid Interface Sci, 492, 207-217.

Batchelor, G. (1967) An Introduction to Fluid Mechanics. Cambridge University Press, Cambridge, 1st edition.

Bradley, A. T., Box, F., Hewitt, I. J. \& Vella, D. (2019) Wettability-independent droplet transport by Bendotaxis. Phys. Rev. Lett., 122(7), 074503.

Butler, M., Box, F., Robert, T. \& Vella, D. (2019) Elasto-capillary adhesion: Effect of deformability on adhesion strength and detachment. Phys. Rev. Fluids, 4(3), 033601.

Carter, W. C. (1988) The forces and behavior of fluids constrained by solids. Acta Metallurgica, 36(8), 2283-2292.

Cho, H., Wu, G., Jolly, J. C., Fortoul, N., He, Z., Gao, Y., Jagota, A. \& Yang, S. (2019) Intrinsically reversible superglues via shape adaptation inspired by snail epiphragm. Proc. Natl Acad. Sci. USA, 116, 13774-13779.

Concus, P. \& Finn, R. (1998) Discontinuous behavior of liquids between parallel and tilted plates. Phys. Fluids, 10, 39-43.

Concus, P., Finn, R. \& McCuan, J. (2001) Liquid Bridges, Edge Blobs, and Scherk-type Capillary Surface. Indiana U. Math. J., 50, 411-441.

de Gennes, P.-G., Brochard-Wyart, F. \& Quéré, D. (2004) Capillarity and Wetting Phenomena: Drops, Bubbles, Pearls, Waves, volume 336. Springer.

Dirks, J.-H. (2014) Physical principles of fluid-mediated insect attachment - Shouldn't insects slip?. Beilstein J. Nanotechnol., 5, 1160-1166.

Dirks, J.-H. \& Federle, W. (2011) Fluid-based adhesion in insects: principles and challenges. Soft Matter, 7, 1104711053.

Geim, A. K., Dubonos, S. V., Grigorieva, I. V., Novoselov, K. S., Zhukov, A. A. \& Shapoval, S. Y. (2003) Microfabricated adhesive mimicking gecko foot-hair. Nat. Mater, 2, 461-463.

Gorb, S. N., Sinha, M., Peressadko, A., Daltorio, K. A. \& Quinn, R. D. (2007) Insects did it first: a micropatterned adhesive tape for robotic applications. Bioinspir. Biomim., 2(4), S117. 
Gorce, J.-B., Hewitt, I. J. \& Vella, D. (2016) Capillary Imbibition into Converging Tubes: Beating Washburn's Law and the Optimal Imbibition of Liquids. Langmuir, 32, 1560-1567.

Hauksbee, F. (1710) An account of an experiment touching the direction of a drop of oil of oranges, between two glass planes, towards any side of them that is nearest press'd together. By Mr. Fr. Hauksbee, F. R. S.. Phil. Trans. R. Soc., 27, 395-396.

Lambert, P. et al. (2013) Surface Tension in Microsystems. Springer.

Leal, L. G. (2007) Advanced Transport Phenomena: Fluid Mechanics and Convective Transport Processes, volume 7. Cambridge University Press.

Lowry, B. J. \& Steen, P. H. (1995) Capillary Surfaces: Stability from Families of Equilibria with Application to the Liquid Bridge. Proc. R. Soc. A, 449, 411-439.

McEwan, A. D. \& Taylor, G. I. (1966) The peeling of a flexible strip attached by a viscous adhesive. J. Fluid Mech., 26, 1-15.

Moriarty, J. A. \& Terrill, E. L. (1996) Mathematical modelling of the motion of hard contact lenses. Eur. J. Appl. Math., 7(6), 575-594.

Renvoisé, P., Bush, J. W. M., Prakash, M. \& Quéré, D. (2009) Drop propulsion in tapered tubes. EPL, 86(6), 64003.

Reyssat, E. (2014) Drops and bubbles in wedges. J. Fluid Mech., 748, 641-662.

Reyssat, E. (2015) Capillary bridges between a plane and a cylindrical wall. J. Fluid Mech., 773, R1.

Ruiz-Gutiérrez, E. \& Ledesma-Aguilar, R. (2020) Lattice-Boltzmann simulations of the dynamics of liquid barrels. J. Phys.: Condens. Matter, 32, 214007.

Ruiz-Gutiérrez, E., Semprebon, C., McHale, G. \& Ledesma-Aguilar, R. (2018) Statics and dynamics of liquid barrels in wedge geometries. J. Fluid Mech., 842, 26-57.

Slater, D. M., Vogel, M. J., Macner, A. M. \& Steen, P. H. (2014) Beetle-inspired adhesion by capillary-bridge arrays: pull-off detachment. J. Adhes. Sci. Technol., 28, 273-289.

Taroni, M. \& Vella, D. (2012) Multiple equilibria in a simple elastocapillary system. J. Fluid Mech., 712, $273-294$.

Trinh, P. H., Wilson, S. K. \& Stone, H. A. (2014) A pinned or free-floating rigid plate on a thin viscous film. $J$. Fluid Mech., 760, 407-430.

Vogel, M. J. \& Steen, P. H. (2010) Capillarity-based switchable adhesion.. Proc. Nat. Acad. Sci. USA, 107, 33773381.

Ward, T. (2011) Capillary-pressure driven adhesion of rigid-planar surfaces. J. Colloid Interface Sci., 354(2), 816824.

\section{A. Full dimensionless equations}

\section{A.1 Free plate motion}

We record here the full equations for the plate and droplet motion while the plate remains free to separate from the substrate and tilt relative to it. First, we note that we can reduce our system of five differentialalgebraic equations (4.9)-(4.11) and (4.13) to four ODEs by eliminating $C$; using (4.9) we have

$$
C=\frac{-\left[(H+\beta X)^{-1}\right]_{\mathrm{drop}}-12 I_{2} \dot{\beta}-12 I_{1}(2 \dot{H}+\beta U)}{12 I_{0}}-H U
$$

To simplify further, we must evaluate the integrals $I_{n}$, defined in (4.12), for $n=0,1,2,3$ and 4; to facilitate this we define the following functions of time only

$$
\psi_{n}(T)=\left\{\begin{array}{ll}
{\left[(H+\beta X)^{n}\right]_{\text {drop }}} & n \neq 0 \\
{[\log (H+\beta X)]_{\text {drop }}} & n=0
\end{array} .\right.
$$


Straightforward calculations give

$$
\begin{gathered}
I_{0}=\frac{-\psi_{-2}}{2 \beta}, \quad I_{1}=\frac{-2 \psi_{-1}+H \psi_{-2}}{2 \beta^{2}}, \quad I_{2}=\frac{2 \psi_{0}+4 H \psi_{-1}-H^{2} \psi_{-2}}{2 \beta^{3}}, \\
I_{3}=\frac{2 \psi_{1}-6 H \psi_{0}-6 H^{2} \psi_{-1}+H^{3} \psi_{-2}}{2 \beta^{4}} \\
I_{4}=\frac{\psi_{2}-8 H \psi_{1}+12 H^{2} \psi_{0}+8 H^{3} \psi_{-1}-H^{4} \psi_{-2}}{2 \beta^{5}}
\end{gathered}
$$

which may then be substituted, along with the value of the shear velocity $U=-\beta^{2} / 4 \psi_{0}$, into the ODEs for the vertical force- and torque-balance to give

$$
\begin{aligned}
\frac{12}{\beta^{4}}\left(\psi_{1}-2 H \psi_{0}-2 \frac{\psi_{0} \psi_{-1}}{\psi_{-2}}-4 H \frac{\psi_{-1}^{2}}{\psi_{-2}}\right) \dot{\beta} & +\frac{12}{\beta^{3}}\left(2 \psi_{0}+4 \frac{\psi_{-1}^{2}}{\psi_{-2}}\right) \dot{H} \\
& =4+2 \frac{\psi_{-1}^{2}}{\beta \psi_{-2}}+6 \frac{\psi_{-1}^{2}}{\psi_{-2} \psi_{0}}
\end{aligned}
$$

and

$$
\begin{aligned}
& \frac{12}{\beta^{5}}\left(\psi_{2}-8 H \psi_{1}+\right.\left.8 H^{2} \psi_{0}+4 \frac{\psi_{0}^{2}}{\psi_{-2}}+16 H \frac{\psi_{0} \psi_{-1}}{\psi_{-2}}+16 H^{2} \frac{\psi_{-1}^{2}}{\psi_{-2}}\right) \dot{\beta} \\
&+\frac{12}{\beta^{4}}\left(4 \psi_{1}-8 H \psi_{0}-8 \frac{\psi_{0} \psi_{-1}}{\psi_{-2}}-16 H \frac{\psi_{-1}^{2}}{\psi_{-2}}\right) \dot{H} \\
&=-2 \frac{\psi_{1}}{\beta^{2}}-4 \frac{\psi_{0} \psi_{-1}}{\beta^{2} \psi_{-2}}-8 H \frac{\psi_{-1}^{2}}{\beta^{2} \psi_{-2}} \\
&+\frac{3}{\beta \psi_{0}}\left(2 \psi_{1}-4 H \psi_{0}-4 \frac{\psi_{0} \psi_{-1}}{\psi_{-2}}-8 H \frac{\psi_{-1}^{2}}{\psi_{-2}}\right)
\end{aligned}
$$

Additionally, we also have equations for the motion of the menisci from (4.13)

$$
\begin{aligned}
\dot{X}_{ \pm} & =-\frac{1}{2\left(H+\beta X_{ \pm}\right)}\left\{\left(\frac{2 \psi_{0}+4 H \psi_{-1}-H^{2} \psi_{-2}}{\beta^{2} \psi_{-2}}+X_{ \pm}^{2}\right) \dot{\beta}\right. \\
& \left.+\left(\frac{-4 \psi_{-1}+2 H \psi_{-2}}{\beta \psi_{-2}}+2 X_{ \pm}\right) \dot{H}+\frac{\beta^{2} \psi_{-1}}{2 \psi_{-2} \psi_{0}}+\frac{\beta \psi_{-1}}{6 \psi_{-2}}\right\}
\end{aligned}
$$

These equations can be solved numerically, given appropriate initial conditions, using MATLAB's ode15s. We use event detection to determine whether either edge of the plate touches the upper substrate; in this case, the plate transitions from these free-plate equations to the equations with solid contact at one edge, which we discuss below.

\section{A.2 Equations after corner contact}

If after some time the plate makes contact at one edge, $H \pm \beta L=0$, then the force balances may be neglected because of the reaction forces (vertical and horizontal) on the plate; we therefore switch 
to solving an equation that expresses torque balance about the contact point, (5.3). Here we focus on deriving the ODEs for the subsequent evolution when the contact occurs at the left edge, so that $H=\beta L$, but the same equations will hold for the other case with the change $L \rightarrow-L$.

Similarly to (A.2), we define the functions

$$
\phi_{n}(T)= \begin{cases}{\left[(L+X)^{n}\right]_{\text {drop }}} & n \neq 0 \\ {[\log (L+X)]_{\text {drop }}} & n=0 .\end{cases}
$$

Evaluating the integrals in (5.2) allows us to evaluate $D$, just as we did for $C$ in (A.1)

$$
D=\frac{2 \phi_{0}}{\phi_{-2}} \dot{\beta}+\frac{\beta^{2} \phi_{-1}}{6 \phi_{-2}} .
$$

We substitute for $D$ using (A.8) in the torque balance (5.4) and meniscus motion equations (5.5) to obtain ODEs for $\beta(T)$ and $X_{ \pm}(T)$

$$
\begin{gathered}
\frac{12}{\beta^{3}}\left(\frac{\phi_{2}}{2}+\frac{2 \phi_{0}^{2}}{\phi_{-2}}\right) \dot{\beta}=2 L-\frac{1}{\beta}\left(\phi_{1}+\frac{2 \phi_{0} \phi_{-1}}{\phi_{-2}}\right) \\
\dot{X}_{ \pm}+\frac{1}{2 \beta\left(L+X_{ \pm}\right)}\left(\left(L+X_{ \pm}\right)^{2}+\frac{2 \phi_{0}}{\phi_{-2}}\right) \dot{\beta}=-\frac{\phi_{-1} \beta}{12 \phi_{-2}\left(L+X_{ \pm}\right)}
\end{gathered}
$$

which are again solved using MATLAB's ode15s with the values of $\beta$ and $X_{ \pm}$immediately prior to contact as initial conditions.

\section{B. Numerical details}

\section{B.1 Fall or stick criteria}

To decide whether the loaded plate ultimately sticks or detaches, we require conditions to terminate the simulations. To this end, we conclude that the plate sticks to the substrate if the separation at the centre of the plate reaches $H=0.2$ and similarly it detaches if the separation gets as high as $H=5$. These values were chosen as they require a significant deviation from the initial plate positions near $H=1$ so it is reasonable to assume the plate motion is consistent from then on. At the same time, these are not too far that the calculations run unnecessarily long or so close to $H=0$ that the droplet is squashed enough to entirely coat the plate. Varying these termination bounds independently does not appear to affect the results of fig. 9 .

\section{B.2 Detecting a meniscus reaching a plate edge}

In practice, the governing equations become singular when the gap width at the droplet meniscus vanishes completely, which can only happen in the contacting regime; this compromises our ability to reach $X_{-}=-L$ in simulations. However, we note from the analysis of Appendix C that, despite this, the droplet reaches the corner in finite time. Moreover, when the capillary-viscous balance of Appendix $\mathrm{C}$ holds, the droplet centre does not migrate sideways and $\beta \omega=\beta\left(X_{+}-X_{-}\right)$remains constant by mass conservation. Therefore a small error in $X_{-}$will only result in a small error in the numerical estimates for $X_{+}$and $\beta$ that are then substituted as the initial conditions for (5.7) that determines the fate of the plate. 
To resolve this, we terminate our numerical solutions when the menisci $X_{ \pm}$reach within a short distance of the corner, in particular when $X_{-}=-0.99 \mathrm{~L}$ or $X_{+}=0.99 \mathrm{~L}$. Once this has occurred then we check the condition (5.7) to decide whether the loading plate ultimately sticks or falls. This should be a reasonable approximation - we expect the values of $\beta$ and $X_{+}+L$ to change by a small amount when running the solution all the way to the corner.

\section{B.3 Sensitivity to initial droplet position}

In calculating fig. 9, we started the simulations with the droplet located centrally on the plate, i.e. with $X_{-}=-X_{+}$so that $\delta X_{+}+\delta X_{-}=0$. If, instead, we started the droplet in the position associated with the growing mode $\sigma_{\circlearrowleft}$ so that the coefficient $B=0$ in (4.21), then the boundaries in fig. 9 do alter a small amount (for example in fig. 9a the stick/fall boundary is further from $\delta H(0)=0$, increasing in magnitude by approximately $10-20 \%$ everywhere). This is perhaps expected, since forcing the initial fastest growing mode should cause the droplet to migrate and the plate to twist faster, accentuating the importance of the twist mechanism when compared to the centred droplet case.

We note that the main conclusions of this study remain the same regardless, but it seems more natural to present results in which the droplet began at the centre.

\section{B.4 Results at small angles and plate lengths}

The results presented in this paper all have initial angles $\beta \geqslant 10^{-2}$, with no result presented where the magnitude of the angle is $\beta=10^{-3}$ or smaller. When the angle is sufficiently small, the presence of high powers of $\beta$ in the ODEs (A.4) and (A.5) (in particular the $\beta^{-5}$ term) mean that the numerical solver cannot meet integration tolerances. One way to avoid this may be to take the small angle approximation further, linearizing all terms $H+\beta X$ with respect to $\beta$ to get simplified ODEs. Since the effect of the tilting perturbation decreases with $\beta(0)$, we do not pursue this here.

Additionally, we did not investigate any case where the droplet meniscus reached the plate edge $\left(X_{ \pm}= \pm L\right)$ before any contact occurred. In this case, we expect the contact line to be pinned at the plate edge and the contact angle to vary instead, with extra physics required to decide how to couple the change in interface curvature at the pinned edge to the flux of incoming fluid from lubrication theory. This scenario seems to occur often when the plate lengths are not much larger than the initial droplet width (the droplets do not have far to migrate to the edge). To avoid the complication of this situation, we have not presented any results with plate half-width $1 / 2<L<1$.

\section{The droplet reaches the corner in finite time}

After the plate has made contact with the substrate, our numerics suggest that the droplet may migrate sufficiently that the meniscus gets close to the corner at $X=-L$. The equations of motion (A.9) \& (A.10) appear to become singular as $X_{-} \rightarrow-L$ (or $X_{+} \rightarrow+L$ if the plate has tilted anticlockwise). A natural question to ask is then: does the droplet reach the corner of the resulting wedge in a finite time and, if so, how long does it take to do so? With a wedge angle that is constant in time, previous work (Reyssat, 2014; Gorce et al., 2016) showed that the leading meniscus does reach the wedge corner in a finite time. Moreover, these studies showed that the leading meniscus propagates at a constant speed as the corner is approached, though this speed is proportional to the wedge angle. In the present problem, the wedge angle is not constant, and so the question then becomes whether its time-dependence modifies the propagation of the droplet significantly? 
To facilitate an analysis that will address this question, we introduce new variables corresponding to the position of the droplet's midpoint (measured with respect to the corner), $\bar{X}$, and the width, $\omega$, of the drop:

$$
\bar{X}=\frac{X_{+}+X_{-}}{2}+L, \quad \omega=X_{+}-X_{-} .
$$

Note that $2 \bar{X}>\omega$ and the left hand meniscus reaches the corner when $2 \bar{X}=\omega$. The functions $\phi_{i}$, given by (A.7), can be written in terms of $\bar{X}$ and $\omega$ by

$$
\begin{array}{ccc}
\phi_{2}=2 \bar{X} \omega, \quad \phi_{1}=\omega, & \phi_{0}=\log \left(\frac{2 \bar{X}+\omega}{2 \bar{X}-\omega}\right), \\
\phi_{-1}=-\frac{4 \omega}{4 \bar{X}^{2}-\omega^{2}}, & \phi_{-2}=-\frac{32 \bar{X} \omega}{\left(4 \bar{X}^{2}-\omega^{2}\right)^{2}} .
\end{array}
$$

Using these expressions, and taking the sum and difference of the two equations defined in (A.10), the equations (A.9) \& (A.10) can be rewritten as

$$
\begin{aligned}
\frac{12}{\beta^{3}} \omega \bar{X}\left[1-\frac{\phi_{0}}{4} \frac{4 \bar{X}^{2}-\omega^{2}}{\bar{X} \omega}\right]\left[1+\frac{\phi_{0}}{4} \frac{4 \bar{X}^{2}-\omega^{2}}{\bar{X} \omega}\right] \dot{\beta} & =2 L-\frac{\omega}{\beta}\left[1+\frac{\phi_{0}}{4} \frac{4 \bar{X}^{2}-\omega^{2}}{\bar{X} \omega}\right] \\
\dot{\bar{X}}+\frac{\bar{X}}{2 \beta}\left[1-\frac{\phi_{0}}{4} \frac{4 \bar{X}^{2}-\omega^{2}}{\bar{X} \omega}\right] \dot{\beta} & =-\frac{\beta}{24} \\
\dot{\omega}+\frac{\omega}{2 \beta}\left[1+\frac{\phi_{0}}{4} \frac{4 \bar{X}^{2}-\omega^{2}}{\bar{X} \omega}\right] \dot{\beta} & =\frac{\beta \omega}{24 \bar{X}} .
\end{aligned}
$$

Note that in eqn. (C.3), if we enforced a constant angle so that $\dot{\beta}=0$ then we recover $\dot{\bar{X}}=-\beta / 24$; in dimensional terms this is $\dot{\bar{x}}=-\bar{\gamma} \alpha / 6 \mu$ which is precisely the result found by Reyssat (2014) for a droplet migrating into a wedge.

Numerical solutions of (A.9) \& (A.10) suggest that the droplet width and plate angle follow scaling laws at late times (see fig. 13a for an example). In particular, the results suggest that $\beta \sim T^{-1 / 3}$ and $\omega \sim T^{+1 / 3}$ with $\bar{X}$ approximately constant (in fig. 13a the variation in $\bar{X}$ is less than $1 \%$ ). If such scalings do indeed hold, then the meniscus will reach the corner in finite time (since $2 \bar{X}-\omega \rightarrow 0$ at a finite $T$ ). The question therefore becomes one of understanding the origin of these scaling laws.

The meniscus may approach the corner in two distinct ways: migration of the whole droplet towards the corner, or squeezing of the droplet as the plate angle decreases. In both scenarios, the capillary component of the torque increases. This is because the capillary pressure at each meniscus is inversely proportional to its distance from the corner, $P=-1 / \beta(X+L)$, so that the capillary torque provided by the droplet $\int(X+L)(-P) \mathrm{d} X$ is $\omega / \beta$; whether migrating at constant angle or squeezing, the droplet width $\omega$ increases as it experiences a narrower gap between the plate and substrate (in the squeezing case the angle $\beta$ also decreases). If this increase in the capillary torque is sufficiently large, we may expect the load torque (which remains constant) to become comparatively negligible, and from then on maintain a balance in eqn. (C.2) between capillary and viscous terms. This observation allows for considerable simplification of (C.2) to

$$
\frac{12}{\beta^{2}} \bar{X}\left[1-\frac{\phi_{0}}{4} \frac{4 \bar{X}^{2}-\omega^{2}}{\bar{X} \omega}\right] \dot{\beta} \approx-1
$$


(a)

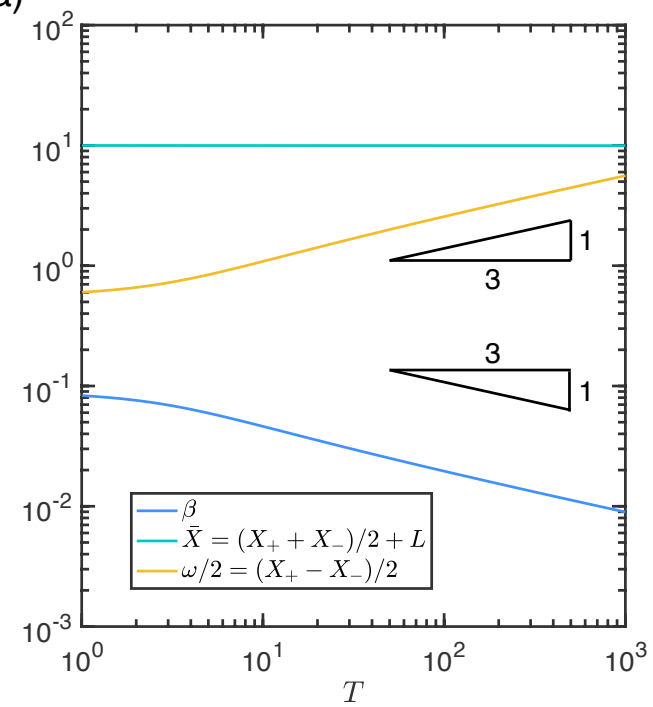

(b)

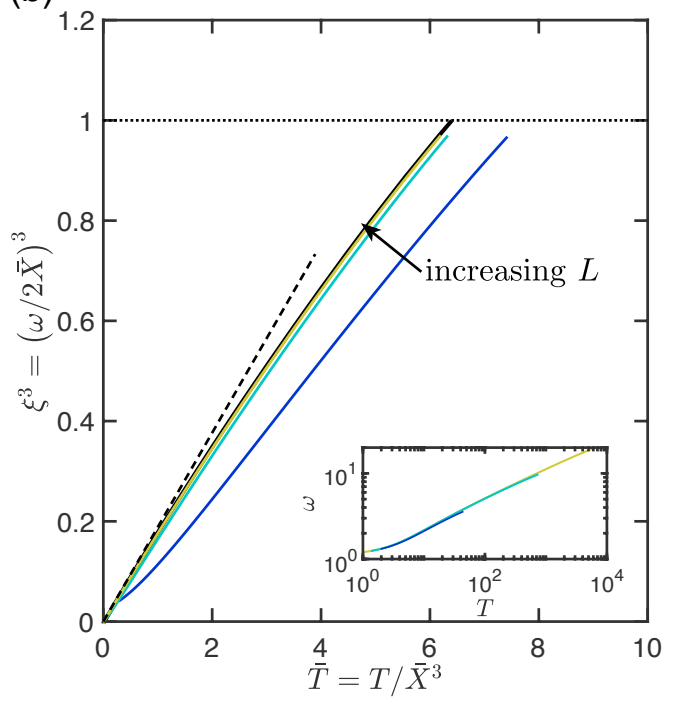

FIG. 13. (a) Evolution of $\beta, \bar{X}, \omega$ in time $T$ after a plate of half-width $L=10$ has made contact with the substrate. At late times, the droplet width $\omega$ and the plate angle $\beta$ appear to follow scaling laws (as indicated), with $\bar{X}$ constant. (These scaling laws are confirmed by the analysis in the main text.) The initial perturbations used were $\beta(0)=10^{-2}, \delta H(0)=-10^{-2}$. (b) Numerical solutions for the droplet width $\omega(T)$ for various plate half-widths $L$ (solid curves) have $\omega^{3}$ increasing approximately linearly in time $T$. These results are compared with the solution to the ODE (C.7) (solid black curve) and the scaling $\omega \sim(3 T / 2)^{1 / 3}$ (dashed line). The droplet reaches the corner when $\xi=\omega / 2 \bar{X}=1$ (dotted line). The solutions shown are for plates of half-width $L=2$ (dark blue), $L=5$ (light green), and $L=10$ (gold). In all cases the initial perturbations were $\beta(0)=10^{-2}, \delta H(0)=-10^{-1}$. Inset: the raw numerical solutions of $\omega(T)$.

This relationship can then be substituted into the droplet motion equations (C.3) \& (C.4), and significantly simplifies the relations for $\bar{X}$ and $\omega$ to:

$$
\dot{\bar{X}} \approx 0, \quad \dot{\omega}+\frac{\omega}{\beta} \dot{\beta} \approx 0 .
$$

The droplet midpoint is fixed, $\bar{X}=$ constant, and the width and angle are inversely related $\omega \propto \beta^{-1}$. More concretely, we know that the droplet volume is fixed, $\beta \bar{X} \omega=1$, and so $\omega(T)=1 /[\bar{X} \beta(T)]$ with $\bar{X}$ constant. It remains to determine $\beta$, or equivalently $\omega$, as a function of time $T$.

Eliminating $\beta$ from (C.5) in favour of $\omega$, and defining $\xi(T)=\omega(T) / 2 \bar{X} \leqslant 1$, we obtain the ODE for $\xi$

$$
\dot{\xi}\left[1-\frac{1-\xi^{2}}{2 \xi} \log \left(\frac{1+\xi}{1-\xi}\right)\right]=\frac{1}{24 \bar{X}^{3}} .
$$

For further insight we note that when the meniscus is far from the corner, $\xi \ll 1, \log [(1+\xi) /(1-\xi)] \sim$ $2 \xi+2 \xi^{3} / 3$ and (C.7) can be simplified to

$$
\xi^{2} \dot{\xi} \sim \frac{1}{16 \bar{X}^{3}} \quad \Longrightarrow \quad \omega \sim\left(\omega_{0}^{3}+\frac{3}{2} T\right)^{1 / 3}, \quad \beta \sim \frac{1}{\bar{X}}\left(\omega_{0}^{3}+\frac{3}{2} T\right)^{-1 / 3}
$$

for some constant $\omega_{0}$, which corresponds to an effective initial droplet width. At late times, we expect that $\omega \sim(3 T / 2)^{1 / 3}$ and $\beta \sim \bar{X}^{-1}(3 T / 2)^{-1 / 3}$. 
The numerical solutions of the full system confirm that $\omega^{3}$ increases approximately linearly with $T$, with examples shown in fig. 13b. The agreement with the scaling relations is relatively good for longer plates; for very short plates there is an offset that we attribute to early-time deviations in $\bar{X}$. In fig. 13a the deviation in $\bar{X}$ once the plate has contacted the substrate is less than $1 \%$, but can be as much as $10 \%$ for the shortest plate shown in fig. 13b $(L=2)$. The plate angle is steeper for short plates, causing a stronger capillary pressure gradient and so the droplet drifts more off-centre before the viscous-capillary balance kicks in. Additionally, the faster migration speeds and shorter distance to travel to the corner may mean that, with shorter plates, we do not reach sufficiently late times for the load torque to be neglected; in these cases, the meniscus still reaches the corner in finite time (as otherwise the load torque eventually becomes negligible) but perhaps not following the scaling as closely as the longer plates.

Note also that the solution of (C.7) is universal, once time is rescaled according to $\tilde{T}=T / \bar{X}^{3}$; the numerical solution of this equation is therefore also plotted in fig. 13b. We find that $\xi \rightarrow 1$ as $\tilde{T} \rightarrow \tilde{T}_{c} \approx 6.4$ which is not far from the estimate made by extrapolating the power-law scaling (valid only for small $\xi$ ): $\omega \rightarrow 2 \bar{X}$ as $T \rightarrow 16 \bar{X}^{3} / 3$. Further, we note that the initial contact often occurs on small timescales in which the droplet will not have moved far, and so we may expect $\bar{X} \approx L$ (especially for longer plates). We conclude that the droplet reaches the corner in a finite time, with an estimate for the time taken given by $T \approx 6.4 \times L^{3}$ (although this appears to be an underestimate for short plates). As a point of comparison, the corresponding time for a droplet to migrate a distance $L$ in a wedge of fixed angle $\beta$ is $T=24 L / \beta$ (Reyssat, 2014); here, the typical wedge angle is $\beta \sim 1 / L$, which suggests a migration time $T \propto L^{2}$ - for long plates, the evolving wedge angle in this problem slows the droplet's motion into the corner compared with the constant angle case.

Finally, we note that the capillary-viscosity balance is valid provided that the torque applied by the load is much smaller than the other terms in eqn. (C.2). From our scaling relations when $\xi=\omega / 2 \bar{X} \ll 1$, we would expect the final term on the right-hand side of (C.2) to look like $\bar{X} T^{2 / 3}$ at late times; if $\bar{X} \approx L$ then the load is negligible at times $T \gg 1$. 